\title{
Optimal On-Off Cooperative Maneuvers for Long-term Satellite Cluster Flight
}

\author{
Leonel Mazal, ${ }^{*}$ Giorgio Mingotti ${ }^{\dagger}$ and Pini Gurfil ${ }^{\ddagger}$ \\ Technion-Israel Institute of Technology, Haifa 32000, Israel
}

\begin{abstract}
When a group of satellites is equipped with a particulary simple propulsion system, e.g. cold-gas thrusters, constraints on the thrust level and total propellant mass renders cluster-keeping extremely challenging. This is even more pronounced in disaggregated space architectures, in which a satellite is formed by clustering a number of heterogenous, free-flying modules. The research described in this paper develops guidance laws aimed at keeping the relative distances between the cluster modules bounded for long mission lifetimes, typically more than a year, while utilizing constant-magnitude low-thrust, with a characteristic on-off profile. A cooperative guidance law capable of cluster establishment and maintenance under realistic environmental perturbations is developed. The guidance law is optimized for fuel consumption, subject to relative distance constraints. Some of the solutions found to the optimal guidance problem require only a single maneuver arc to keep the cluster within relatively close distances for an entire year.
\end{abstract}

\footnotetext{
${ }^{*}$ Doctoral Student, Distributed Space Systems Lab, Faculty of Aerospace Engineering. email: leomazal@techunix.technion.ac.il

${ }^{\dagger}$ Post-Doctoral Research Associate, Distributed Space Systems Lab, Faculty of Aerospace Engineering. email: mingotti@techunix.technion.ac.il

${ }^{\ddagger}$ Associate Professor, Distributed Space Systems Lab, Faculty of Aerospace Engineering, Associate Fellow AIAA. email: pgurfil@technion.ac.il
} 


\section{Nomenclature}

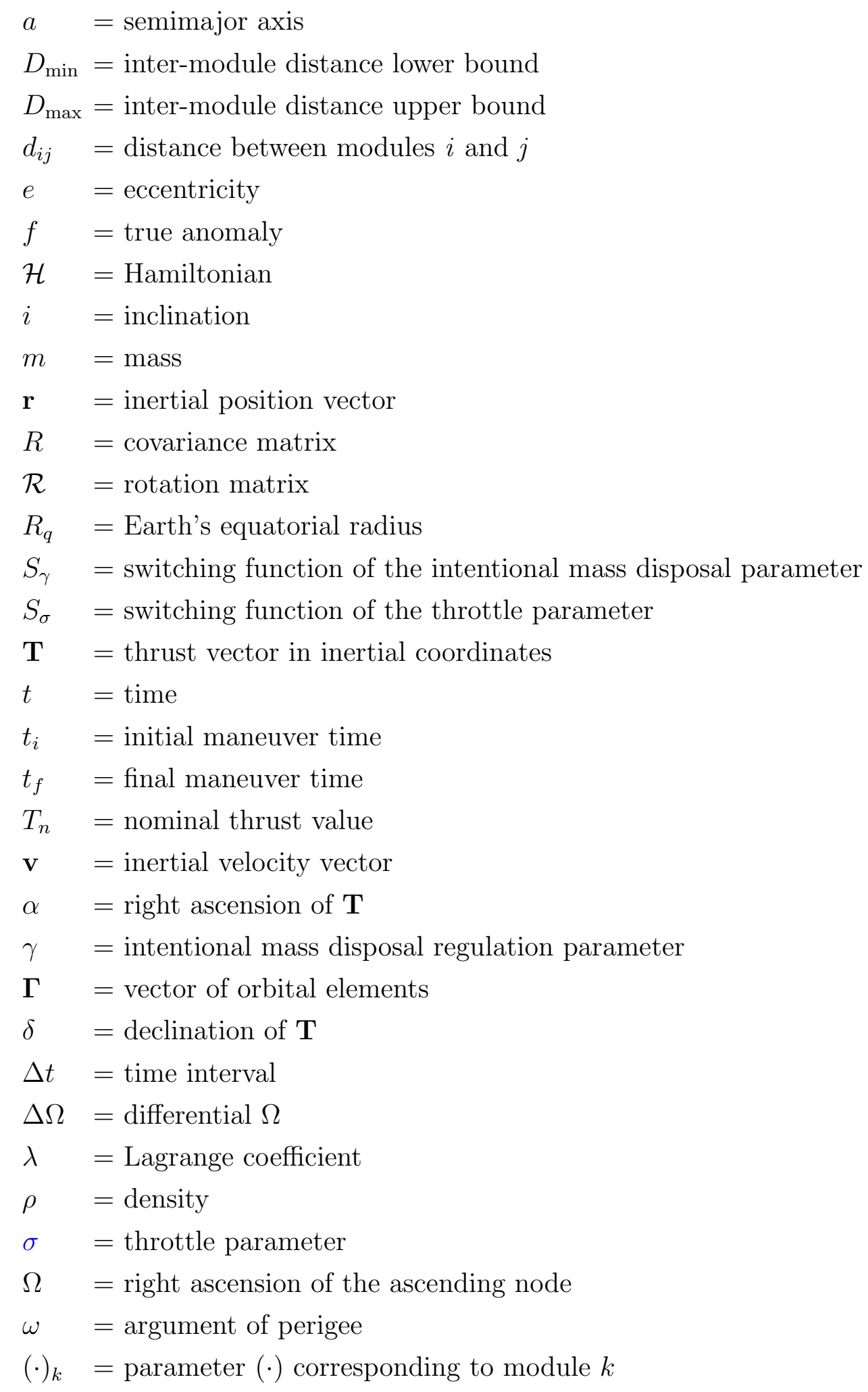




\section{Introduction}

Disaggregated space architectures (DSA) constitute an emerging concept in the realm of distributed space systems. The main idea is to replace monolithic satellites by multiple freeflying, physically-separated modules interacting through wireless cross-links. These disaggregated satellite modules (DSM) can be heterogeneous, having one or more pre-determined functions, e.g. navigation, attitude control, power generation and payload operation. DSA enable extended spacecraft operability; for instance, a failed DSM can be replaced, whereas in a monolithic satellite a failed subsystem might cause premature mission termination.

Unlike satellite formation flying missions, the DSM do not have to operate in a tightlycontrolled formation; instead, they are required to maintain the inter-module distances bounded (typically between $100 \mathrm{~m}$ and $100 \mathrm{~km}$ ) for the entire mission lifetime. This concept is termed cluster flight. Some of the main ideas which DSA rely upon were formally introduced by Brown and Eremenko [1]. Later works discussed the advantages and disadvantages of DSA compared to conventional architectures [2].

Cluster flight is challenging, because without control forces initially-close DSM will tend to drift apart due to differential accelerations. Since the total propellant on each DSM is strictly limited, and the propulsion system is of limited efficiency (e.g. cold-gas), keeping a cluster of DSM in prescribed maximum and minimum distances while maintaining at least one of the DSM (e.g., the payload module) on a given reference orbit becomes a challenging problem. This problem couples high-fidelity astrodynamical modeling, guidance, and orbit control.

If Keplerian dynamics are assumed, equal semimajor axes guarantee periodic relative motion [3]. However, in realistic scenarios the problem is much more complicated. The most significant perturbations affecting low Earth-orbit satellites are the Earth oblateness and drag. Many works presented strategies for mitigating the relative drifts among satellites subject to the main perturbations. Constraints leading to $J_{2}$ secular effect mitigation and concomitant optimal multi-impulsive maneuvers for spacecraft formation flying were pre- 
viously proposed [4-9]. An extension of perturbation mitigation for the problem of cluster flight was recently developed [10], wherein constraints on the relative states, as well as an impulsive cluster-keeping algorithm leading to bounded motion in presence of zonal harmonics and drag, were developed.

Impulsive velocity changes, while frequently providing useful approximations, do not represent realistic maneuvers, which are continuous processes. In this context, several works dealt with time-continuous maneuvers for distributed space systems. Most of these works derived control laws with a continuously-variable thrust magnitude [11-13].

Cold-gas propulsion systems are useful for disaggregated spacecraft due to simplicity and low cost. However, a significant drawback is that the magnitude of the exerted thrust cannot be easily regulated. This feature leads to control profiles known as on-off. Attempts at finding optimal trajectories for satellites, while assuming constant thrust levels, were previously made $[14,15]$. If the problem is well formulated, optimization theory can lead to cooperative optimal guidance laws for a cluster of DSM. It is desirable to design cooperative maneuvers since they tend to minimize the global propellant consumption while balancing the consumed propellant among the satellites.

A critical fact to consider concerns the ballistic coefficients of the DSM. In the presence of drag, differences in ballistic coefficients may cause rapid drift among the DSM. Consequently, any cluster-keeping strategy should consider balancing the ballistic coefficients after any maneuver. This led to the idea of intentional mass disposal [10].

In this paper, an optimal control approach is developed that leads to cooperative propellantoptimal guidance laws steering a cluster of DSM from given initial conditions to terminal conditions, which mitigate the relative drifts while keeping the ballistic coefficients balanced. The DSM are assumed to have the same cross-sectional area and drag coefficient. Therefore, to keep the ballistic coefficients equal, the developed maneuvers aim to match the masses at the end of each maneuver. Since the thrust magnitude is constant, on-off maneuvers are designed. The proposed guidance law can keep a cluster of DSM in a bounded configuration for very long time scales - possibly for the entire mission - and is hence quite different from 
similar works dedicated to spacecraft formation flying.

\section{Strategy for Cluster Flight}

Consider a cluster of $N$ modules, $\mathrm{DSM}_{k}, k=1 \ldots N$. At any time $t$, it is required to hold the inter-module distances $d_{i j}(t)(i, j=1,2, \ldots, N, i \neq j)$ between lower and upper bounds, $D_{\min }$ and $D_{\max }$, respectively:

$$
D_{\min } \leq d_{i j}(t)=\left\|\mathbf{r}_{i}(t)-\mathbf{r}_{j}(t)\right\| \leq D_{\max }
$$

where $\mathbf{r}_{i}$ and $\mathbf{r}_{j}$ denote the position vectors of $\mathrm{DSM}_{i}$ and $\mathrm{DSM}_{j}$, respectively, in an inertial reference frame. Generally speaking, the upper bound $D_{\max }$ is related to the communication cross-links between the DSM, while the lower bound $D_{\min }$ is imposed for collision avoidance.

Considering possible mission specifications, additional constraints may be posed on the orbit of the payload module, by requiring, for example,

$$
a_{\min } \leq a_{1}(t) \leq a_{\max }, \quad e_{\min } \leq e_{1}(t) \leq e_{\max }, \quad i_{\min } \leq i_{1}(t) \leq i_{\max }
$$

where $a$ denotes the semimajor axis, $e$ is the eccentricity, and $i$ is the inclination. The subindex $(\cdot)_{1}$ denotes the orbital element $(\cdot)$ referred to the payload, and the sub-indices $(\cdot)_{\min }$ and $(\cdot)_{\max }$ denote lower and upper bounds respectively, for the orbital element $(\cdot)$. Notice that in this formulation the orbital elements $a_{1}(t), e_{1}(t)$, and $i_{1}(t)$ are osculating elements. Therefore, the upper and lower bounds should be set such that they account for the natural oscillations of the elements, and such that Eq. (2) is inline with mission specifications.

This work proposes a general strategy for cluster establishment and cluster-keeping, which consists of performing a cooperative establishment maneuver at the beginning of the mission, and then performing further cooperative re-establishment maneuvers at any later time upon necessity. By necessity it is meant that, at time $t$, the constraints Eq. (1) or Eq. (2) are

violated. The flowchart in Fig. 1 illustrates the idea. In this figure, $t_{\text {end-mission }}$ denotes the 


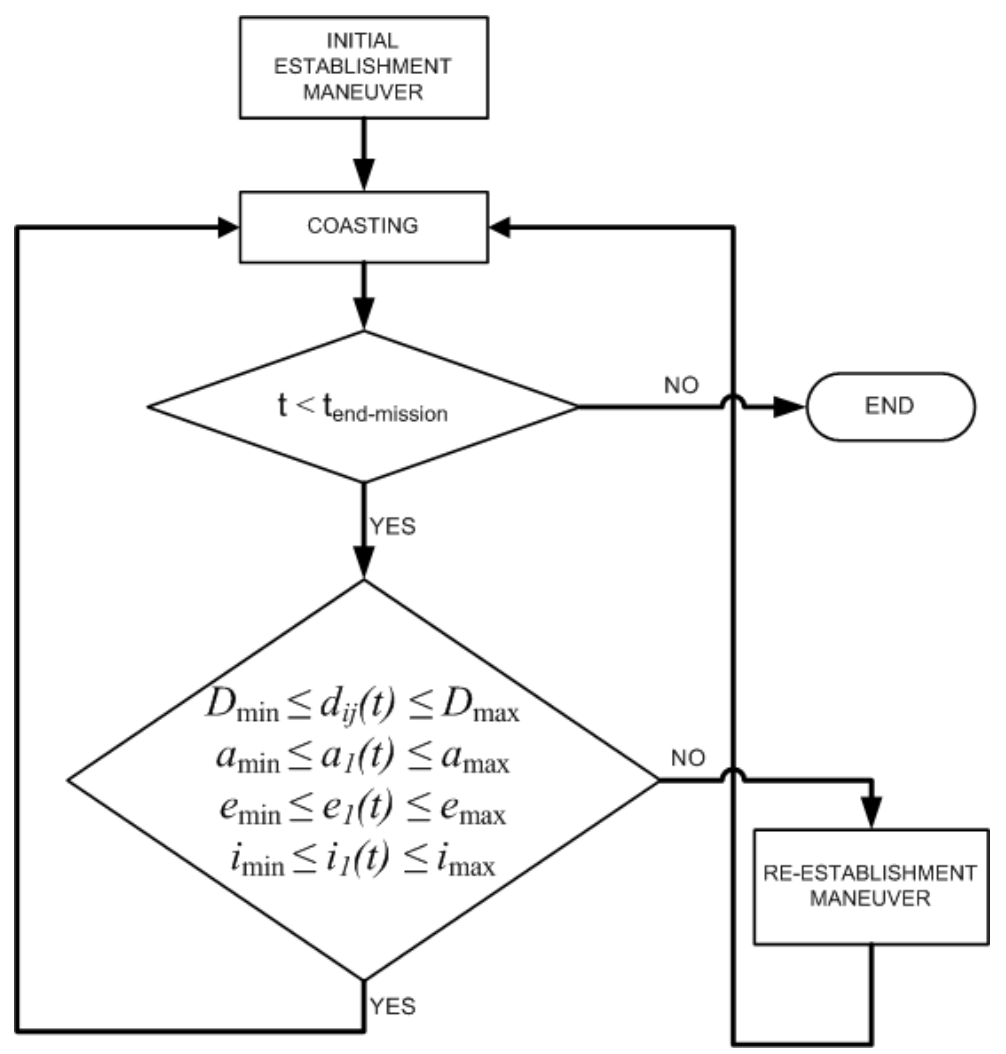

Figure 1. Flow chart of the global cluster-keeping scheme.

final time of the entire mission.

In light of the aforementioned strategy, it is important that each establishment (or reestablishment) maneuver steers the system to a set of states that enable the system to remain as long as possible within the given constraints, so as to reduce the number of maneuvers required during the mission lifetime.

The states attained at the end of each cooperative maneuver are referred to as terminal states. The main goal of this work is to derive an optimal guidance method yielding cooperative maneuvers aimed at reaching specific terminal states, which satisfy constraints that enable a cluster of a given number of DSM to (i) hold the inter-module distances between given bounds for long time intervals, and (ii) track a given reference orbit (at least one module) up to some prescribed accuracy. These maneuvers must be optimal in terms of the consumed propellant, and enable the cluster to coast for long time intervals without corrective maneuvers. 
Consequently, the next step is to formulate the said problem as an optimal guidance problem.

\section{Optimal Guidance Problem Formulation}

\section{A. Bounded-Motion Constraints}

In Ref. [10], constraints on the relative states of the satellites were found, for which the resulting inter-module distance is lower- and upper-bounded. Expressions for these bounds were provided. The perturbations for which the constraints were developed included zonal harmonics and drag, assuming that the distribution of density is time-invariant and symmetric with respect to the Earth's polar axis. Another assumption was that the ballistic coefficients of the modules are equal and time-invariant. In case of $N$ modules, this is stated as

$$
\frac{C_{D_{k}} S_{k}}{m_{k}}=\frac{C_{D_{l}} S_{l}}{m_{l}}=\text { const. } \quad k, l=1, \ldots, N
$$

where $C_{D_{k}}, m_{k}$, and $S_{k}$ denote the respective drag coefficient, mass, and cross-sectional area (normal to the velocity direction) of $\mathrm{DSM}_{k}$.

Define an Earth-centered inertial (ECI) frame, with the fundamental plane lying on the equatorial plane, the $\hat{\mathbf{x}}$ axis pointing towards the mean vernal equinox of epoch, the $\hat{\mathbf{z}}$ axis pointing towards the mean rotational axis of epoch, and $\hat{\mathbf{y}} \triangleq \hat{\mathbf{z}} \times \hat{\mathbf{x}}$. In this frame,

$\mathbf{r}_{k} \triangleq\left[\begin{array}{lll}x_{k} & y_{k} & z_{k}\end{array}\right]^{\top}$ and $\mathbf{v}_{k}=\left[\begin{array}{lll}v x_{k} & v y_{k} & v z_{k}\end{array}\right]^{\top} \triangleq\left[\begin{array}{lll}\dot{x}_{k} & \dot{y}_{k} & \dot{z}_{k}\end{array}\right]^{\top}$ denote the respective position and velocity vectors of $\mathrm{DSM}_{k}$. Define

$$
\Gamma_{k} \triangleq\left[a_{k}, e_{k}, i_{k}, \omega_{k}, f_{k}, \Omega_{k}\right]^{\top}
$$

where $a_{k}$ is the semi-major axis, $e_{k}$ is the eccentricity, $i_{k}$ is the inclination, $\omega_{k}$ is the argument of perigee, $f_{k}$ is the true anomaly, and $\Omega_{k}$ denotes the right ascension of the ascending node (RAAN), all corresponding to $\mathrm{DSM}_{k}$, with $k=1, \ldots, N$. If, at a given time $t=t^{*}$, the state vector of $\mathrm{DSM}_{k}$ is such that 

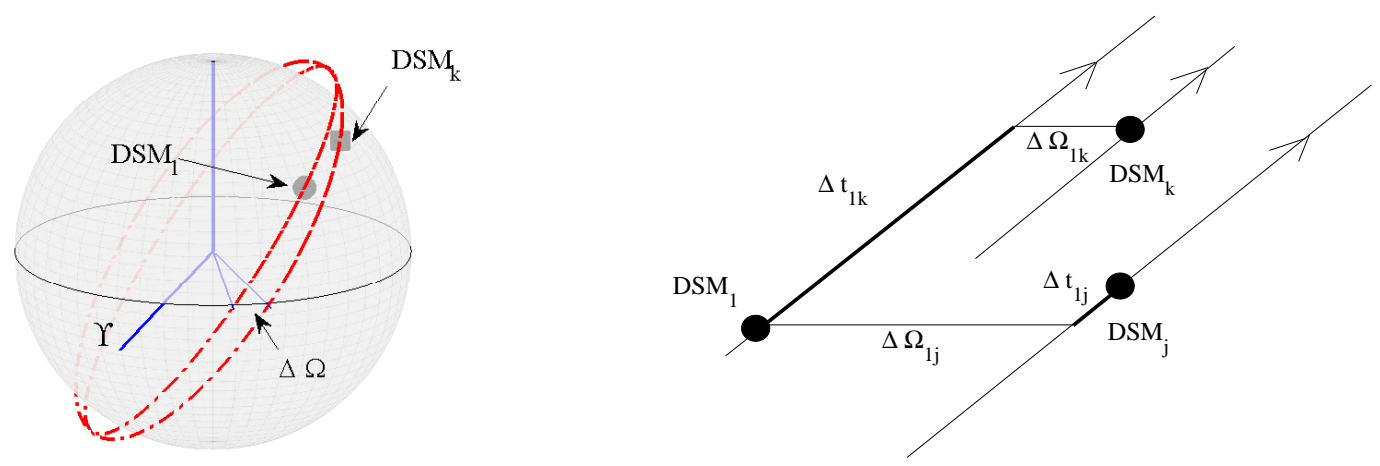

(a) Schematic view of the orbit geometry.

(b) Schematic view of the constraints in Eqs. (5).

Figure 2. Final constraints on the relative states.

$$
\boldsymbol{\Gamma}_{k}\left(t^{*}\right)=\boldsymbol{\Gamma}_{1}\left(t^{*}+\Delta t_{1 k}\right)+\left[\begin{array}{c}
\mathbf{0}_{5 \times 1} \\
\Delta \Omega_{1 k}
\end{array}\right], \quad k=2,3, \ldots, N
$$

where $\Delta t_{1 k}$ denotes a shift in time and $\Delta \Omega_{1 k}$ represents a shift in the RAAN, then the distance between $\mathrm{DSM}_{1}$ and $\mathrm{DSM}_{k}$, denoted by $d_{1 k}(t)$, is bounded by [10]

$$
2 \eta_{\min } \sin \left(\frac{\left|\Delta \Omega_{1 k}\right|}{2}\right)-V_{\max }\left|\Delta t_{1 k}\right| \leq d_{1 k}(t) \leq V_{\max }\left|\Delta t_{1 k}\right|+2 \eta_{\max } \sin \left(\frac{\left|\Delta \Omega_{1 k}\right|}{2}\right) \forall t \geq t^{*}, k=2,3 \ldots, N
$$

$\Delta t_{1 k}$ and $\Delta \Omega_{1 k}$ are both user-defined parameters. In addition, $V_{\max } \triangleq \max _{t}\left\|\mathbf{v}_{1}(t)\right\|$,

$$
\eta_{1}(t) \triangleq \sqrt{x_{1}(t)^{2}+y_{1}(t)^{2}}
$$

and $\eta_{\max } \triangleq \max _{t} \eta_{1}(t), \eta_{\min } \triangleq \min _{t} \eta_{1}(t)$.

Fig. 2 illustrates the relative geometries generated by Eq. (5). Moreover, if

$$
\boldsymbol{\Gamma}_{l}\left(t^{*}\right)=\boldsymbol{\Gamma}_{1}\left(t^{*}+\Delta t_{1 l}\right)+\left[\begin{array}{c}
\mathbf{0}_{5 \times 1} \\
\Delta \Omega_{1 l}
\end{array}\right], \quad l \neq k
$$


then the distance between $\mathrm{DSM}_{k}$ and $\mathrm{DSM}_{l}$, denoted by $d_{k l}(t)$, satisfies

$$
2 \eta_{\min } \sin \left(\frac{\left|\Delta \Omega_{k l}\right|}{2}\right)-V_{\max }\left|\Delta t_{k l}\right| \leq d_{k l}(t) \leq V_{\max }\left|\Delta t_{k l}\right|+2 \eta_{\max } \sin \left(\frac{\left|\Delta \Omega_{k l}\right|}{2}\right) \forall t \geq t^{*}, l \neq k
$$

where $\Delta \Omega_{k l} \triangleq \Delta \Omega_{1 l}-\Delta \Omega_{1 k}$ and $\Delta t_{k l} \triangleq \Delta t_{1 l}-\Delta t_{1 k}$.

To accurately determine the values of the bounds (6) and (9) one should compute $V_{\max }$, $\eta_{\max }$, and $\eta_{\min }$, which means that the orbit of $\mathrm{DSM}_{1}$ in Eqs. (6) should be known.

For full astrodynamical models the obtained theoretical bounds are not strictly valid. It may happen that at a certain time $t$ the distance between the modules exceeds the established bounds. However, in real missions, the advantage of using Eq. (5) is that these conditions can significantly mitigate the relative drift between the DSM, even in a real space environment, as shown in Ref. [10]. Hence, the guidance law developed herein pursues to achieve these constraints.

\section{B. Thruster Configuration}

It is assumed that the DSM are equipped with four identical thrusters, and that the thrust magnitude is constant, i.e. the operation profile for each thruster is on-off. Real thrusters are usually characterized by misalignment, timing errors and magnitude uncertainty. However, to formulate a design model, these errors are neglected. In order to cope with the aforementioned undesired errors and uncertainties, closed-loop control strategies should be used. A further discussion of this subject is provided in the sequel.

The array of engines is comprised of two pairs of opposite thrusters. The axes of the two pairs are orthogonal (see Fig. $3(\mathrm{a}))^{1}$. In a body frame $\mathcal{B}$, which axes are aligned with the thruster axes, the direction of thrust generated by the $m^{\text {th }}$ thruster, denoted by $\mathbf{b}_{i}^{\mathcal{B}}, i=$

\footnotetext{
${ }^{1}$ The same approach could be implemented for three orthogonal pairs of opposite thrusters. However, in small-satellite missions, two pairs may be sufficient for orbit keeping.
} 
$1,2,3,4$, is given by the following vectors:

$$
\mathbf{b}_{1}^{\mathcal{B}} \triangleq\left[\begin{array}{l}
1 \\
0 \\
0
\end{array}\right] \quad \mathbf{b}_{2}^{\mathcal{B}} \triangleq\left[\begin{array}{c}
-1 \\
0 \\
0
\end{array}\right] \quad \mathbf{b}_{3}^{\mathcal{B}} \triangleq\left[\begin{array}{l}
0 \\
1 \\
0
\end{array}\right] \quad \mathbf{b}_{4}^{\mathcal{B}} \triangleq\left[\begin{array}{c}
0 \\
-1 \\
0
\end{array}\right]
$$

When a thruster is activated, it exerts a thrust of constant nominal magnitude $T_{n}$. Thus, the thrust vector in body axes is given by

$$
\mathbf{T}^{\mathcal{B}}=T_{n}\left[\begin{array}{c}
\kappa_{1}-\kappa_{2} \\
\kappa_{3}-\kappa_{4} \\
0
\end{array}\right]
$$

where $\kappa_{m} \in\{0,1\}$. If $\kappa_{m}(t)=1$, then at time $t$ the $m^{\text {th }}$ thruster is being fired, whereas if $\kappa_{m}(t)=0$ then at time $t$ the $m^{\text {th }}$ thruster is off. The mass flow rate of the $k^{\text {th }}$ satellite, $\dot{m}_{k}$, is given by

$$
\dot{m}_{k}=-\frac{T_{n}}{I_{s p} g_{0}} \sum_{m=1}^{4} \kappa_{m_{k}}
$$

where $I_{s p}$ is the specific impulse and $g_{0}$ is the standard gravity acceleration at sea level.

This configuration allows for zero-thrust mass consumption, which may be necessary for balancing the ballistic coefficients as previously mentioned. Whether it is necessary to intentionally dispose mass or not will be determined by the dynamic optimization procedure.

\section{Fictitious Dual-Engine Configuration}

In order to facilitate the formulation of the optimization problem, the physical configuration of the engines, given by Eqs. (11) and (12), is replaced by a fictitious dual-engine configuration, as illustrated in Fig. 3(b). This configuration consists of two engines labeled E1 and E2. $E 1$ is used for controlling the orbital motion, exerting the required thrust while consuming propellant; at most, a single thruster per pair is allowed to work. On the other 


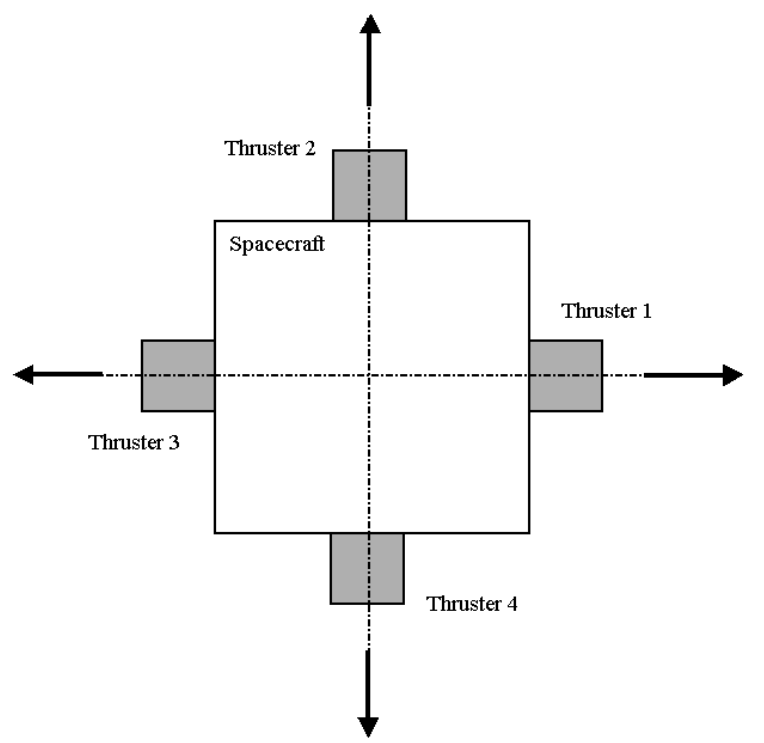

(a) Thruster configuration.

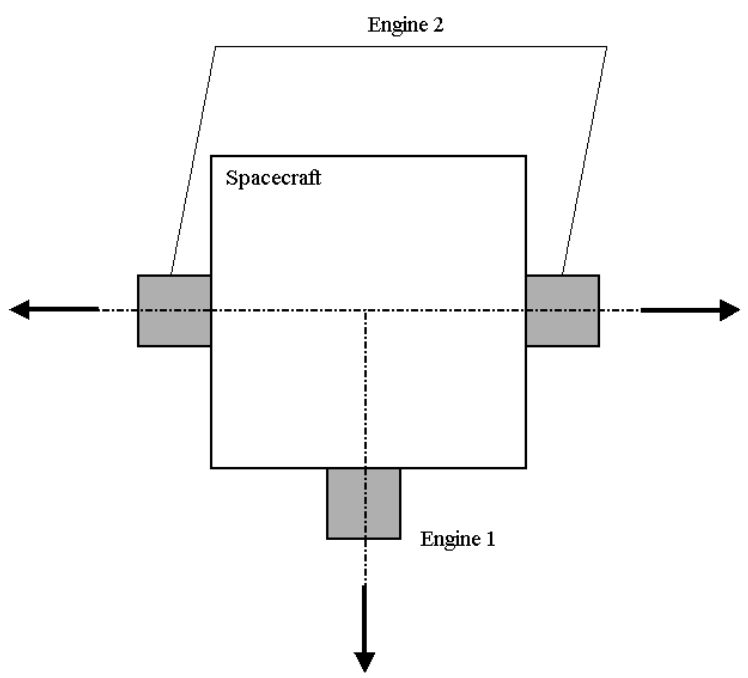

(b) Fictitious dual-engine configuration.

Figure 3. Thruster configuration.

hand, $E 2$ is used to burn fuel and to control the intentional mass disposal without exerting thrust, meaning that both thrusters in a pair are fired simultaneously.

For each DSM, it is possible to define several thrusting modes. Table 1 details the maximum level of effective thrust available, the concomitant mass consumption, and the maximum available level of intentional mass disposal for 5 possible operation modes. In Table $1, T_{k}$ stands for the thrust level for engine $E 1$ (orbital motion control), $C_{k}$ represents its corresponding mass consumption, and $D_{k}$ represents the mass disposal of engine $E 2$. The total mass consumption is given by the following relationship:

$$
\dot{m}_{k}=-\sigma_{k} \frac{C_{k}}{I_{s p} g_{0}}-\gamma_{k} \frac{D_{k}}{I_{s p} g_{0}}
$$

where $\sigma_{k}$ represents the throttle parameter that must be either 0 or 1 , and $\gamma_{k}$ is a parameter regulating the intentional mass disposal of $\mathrm{DSM}_{k}$, which must be either 0 or 1 .

In Mode I, one thruster for each pair is on duty, with no intentional mass disposal. In contrast, when Modes II and III are selected, no effective thrust is possible, because either none of the thrusters are active or both of them are working simultaneously. Mode IV 
Table 1. Spacecraft thrusting modes.

\begin{tabular}{|c|c|c|c|c|}
\hline Mode & Description & $T_{k}$ & $C_{k}$ & $D_{k}$ \\
\hline I & $\begin{array}{c}\text { Two orthogonal thrusters, } \\
\text { one per pair, can be simultaneously } \\
\text { fired }\end{array}$ & $\sqrt{2} T_{n}$ & $2 T_{n}$ & 0 \\
\hline II & $\begin{array}{l}\text { Both thrusters of one pair } \\
\text { can be simultaneously fired }\end{array}$ & 0 & 0 & $2 T_{n}$ \\
\hline III & $\begin{array}{l}\text { Both thrusters of both pairs } \\
\text { can be simultaneously fired }\end{array}$ & 0 & 0 & $4 T_{n}$ \\
\hline IV & $\begin{array}{l}\text { One thruster of one pair can be fired, } \\
\text { and/or both thrusters (simultaneously) } \\
\text { of the other pair can be fired }\end{array}$ & $T_{n}$ & $T_{n}$ & $2 T_{n}$ \\
\hline V & $\begin{array}{l}\text { One thruster of one pair } \\
\text { can be fired }\end{array}$ & $T_{n}$ & $T_{n}$ & 0 \\
\hline
\end{tabular}

represents a hybrid configuration in which both orbital control and intentional mass disposal are used. In the present paper, Mode IV is pre-selected for all the satellites. Finally, in Mode $\mathrm{V}$ a single thruster is allowed to exert thrust, but no intentional mass disposal is possible.

For engine $E 1$, a spherical-coordinate representation of the thrust vector is adopted with respect to the ECI frame, so that

$$
\mathbf{T}_{k}=\sigma_{k} T_{k}\left[\cos \left(\alpha_{k}\right) \cos \left(\delta_{k}\right), \sin \left(\alpha_{k}\right) \cos \left(\delta_{k}\right), \sin \left(\delta_{k}\right)\right]^{\top}
$$

where $\alpha_{k}$ and $\delta_{k}$ denote the right ascension and declination angles respectively, all corresponding to $\mathrm{DSM}_{k}$. 


\section{Design Model}

The dynamical model used for solving the optimization problem stated in the next sections is given by

$$
\begin{aligned}
\dot{\mathbf{r}}_{k} & =\mathbf{v}_{k} \\
\dot{\mathbf{v}}_{k} & =-\mu \frac{\mathbf{r}_{k}}{\left\|\mathbf{r}_{k}\right\|^{3}}-\frac{\mu J_{2} R_{q}^{2}}{2\left\|\mathbf{r}_{k}\right\|^{5}}\left(6\left[\begin{array}{c}
0 \\
0 \\
z_{k}
\end{array}\right]+\left[3-15\left(\frac{z_{k}}{\left\|\mathbf{r}_{k}\right\|}\right)^{2}\right] \mathbf{r}_{k}\right)-\rho\left\|\mathbf{v}_{k}\right\| \frac{S C_{D}}{2 m_{k}} \mathbf{v}_{k}+\frac{\mathbf{T}_{k}}{m_{k}} \\
\dot{m}_{k} & =-\sigma_{k} \frac{C_{k}}{I_{s p} g_{0}}-\gamma_{k} \frac{D_{k}}{I_{s p} g_{0}}
\end{aligned}
$$

where $m_{k}$ denotes the instantaneous mass of $\mathrm{DSM}_{k}$. This model includes Keplerian dynamics

perturbed by $J_{2}$, (the first term of the zonal harmonics potential), drag and thrust. In Eqs. (15) the density $\rho$ is assumed constant. However, this assumption will be removed later on, and the performance will be evaluated using high-fidelity simulations. The variable $\mu$ denotes the gravitational parameter.

\section{E. Optimal Guidance Problem}

Define the state vector of each DSM, at time $t$, as

$$
\mathbf{y}_{k}(t)=\left[x_{k}(t), y_{k}(t), z_{k}(t), v x_{k}(t), v y_{k}(t), v z_{k}(t), m_{k}(t)\right]^{\top}
$$

The optimal guidance problem can now be explicitly formulated: Assume that the initial conditions at $t=t_{i}$ are $\mathbf{y}_{k}\left(t_{i}\right), k=1,2, \ldots N$. Define the cost functional

$$
\mathcal{J}=\int_{t_{i}}^{t_{f}} \sum_{k=1}^{N}\left(\sigma_{k} C_{k}+\gamma_{k} D_{k}\right) \mathrm{dt}
$$

which represents the total mass consumption (up to scaling by the constant term $I_{s p} g_{0}$ ). Recall that $\alpha_{k}$ and $\delta_{k}$ are the steering angles of the thrust vector $\mathbf{T}_{k}$, and that $\sigma_{k}$ and $\gamma_{k}$ are the throttle parameters related to the control thrust and the intentional mass disposal, respectively. Derive a cooperative guidance law, determined by the control parameters 
$\left\{\sigma_{k}(t), \gamma_{k}(t) ; \alpha_{k}(t), \delta_{k}(t)\right\}, k=1,2, \ldots, N$, that steers the cluster of DSM from $\mathbf{y}_{k}\left(t_{i}\right)$ to the terminal states $\mathbf{y}_{k}\left(t_{f}\right)$, such that $\mathcal{J}$ is minimized, and the following equality constraints are satisfied:

$$
\begin{gathered}
a_{1}\left(t_{f}\right)=-\frac{\mu\left\|\mathbf{r}_{1}\left(t_{f}\right)\right\|}{\left\|\mathbf{v}_{1}\left(t_{f}\right)\right\|^{2}\left\|\mathbf{r}_{1}\left(t_{f}\right)\right\|-2 \mu}=a_{\mathcal{D}} \\
e_{1}\left(t_{f}\right)=\sqrt{1+\frac{\left\|\mathbf{r}_{1}\left(t_{f}\right) \times \mathbf{v}_{1}\left(t_{f}\right)\right\|^{2}}{\mu^{2}\left\|\mathbf{r}_{1}\left(t_{f}\right)\right\|}\left(\left\|\mathbf{v}_{1}\left(t_{f}\right)\right\|^{2}\left\|\mathbf{r}_{1}\left(t_{f}\right)\right\|-2 \mu\right)}=e_{\mathcal{D}} \\
i_{1}\left(t_{f}\right)=\arccos \left(\frac{x_{1}\left(t_{f}\right) v y_{1}\left(t_{f}\right)-y_{1}\left(t_{f}\right) v x_{1}\left(t_{f}\right)}{\left\|\mathbf{r}_{1}\left(t_{f}\right) \times \mathbf{v}_{1}\left(t_{f}\right)\right\|}\right)=i_{\mathcal{D}} \\
\mathbf{r}_{k}\left(t_{f}\right)=\mathcal{R}\left(\Delta \Omega_{1 k}\right)\left[\mathbf{r}_{1}\left(t_{f}\right)+\mathbf{v}_{1}\left(t_{f}\right) \Delta t_{1 l}-\frac{\mu}{2} \frac{\mathbf{r}_{1}\left(t_{f}\right)}{\left\|\mathbf{r}_{1}\left(t_{f}\right)\right\|^{3}} \Delta t_{1 k}^{2}\right] \quad k=2,3, \ldots, N \\
\mathbf{v}_{k}\left(t_{f}\right)=\mathcal{R}\left(\Delta \Omega_{1 k}\right)\left[\mathbf{v}_{1}\left(t_{f}\right)-\mu \frac{\mathbf{r}_{1}\left(t_{f}\right)}{\left\|\mathbf{r}_{1}\left(t_{f}\right)\right\|^{3}} \Delta t_{1 k}\right] \quad k=2,3, \ldots, N
\end{gathered}
$$

and

$$
m_{k}\left(t_{f}\right)=m_{1}\left(t_{f}\right) \quad k=2,3, \ldots, N
$$

where, recalling Eq. (2),

$$
a_{\mathcal{D}} \triangleq \frac{a_{\max }+a_{\min }}{2}, \quad e_{\mathcal{D}} \triangleq \frac{e_{\max }+e_{\min }}{2}, \quad i_{\mathcal{D}} \triangleq \frac{i_{\max }+i_{\min }}{2}
$$

and $\mathcal{R}\left(\Delta \Omega_{1 k}\right)$ is given by

$$
\mathcal{R}\left(\Delta \Omega_{1 k}\right) \triangleq\left[\begin{array}{ccc}
\cos \left(\Delta \Omega_{1 k}\right) & -\sin \left(\Delta \Omega_{1 k}\right) & 0 \\
\sin \left(\Delta \Omega_{1 k}\right) & \cos \left(\Delta \Omega_{1 k}\right) & 0 \\
0 & 0 & 1
\end{array}\right] \quad k=2,3, \ldots, N
$$

Eqs. (18) require $\mathrm{DSM}_{1}$ to track a reference orbit. These terminal constraints allow the payload to track a specific orbit during the post-maneuver coasting arc, so as to satisfy possible mission requirements, until Eq. (2) is violated. On the other hand, Eqs. (19) represent an approximation of the constraints given by Eq. (5). In light of the discussion presented in 
Section A, it is desirable to guide the DSM to states satisfying Eq. (5). However, to strictly apply constraints $(5)$, one should forward integrate the real trajectory of $\mathrm{DSM}_{1}$, from $t=t_{f}$ to $t=t_{f}+\Delta t$. However, in order to be able to formulate and solve the optimal guidance problem, the integration is replaced by Eqs. (19), which assume that during the interval of time from $t=t_{f}$ to $t=t_{f}+\Delta t, \mathrm{DSM}_{1}$ follows a trajectory arc with a constant acceleration given by $-\mu \mathbf{r}_{1}\left(t_{f}\right) /\left\|\mathbf{r}_{1}\left(t_{f}\right)\right\|^{3}$. For $\Delta t$ of a few seconds, as used in this work, this represents a good approximation, as will be seen in a numerical example of Section VI. The purpose of imposing the constraints (19) is to achieve a slow relative post-maneuver drift. Finally, the constraints (20) lead to the equalities of the post-maneuver masses. Since it was assumed that the cross-sectional areas as well as the drag coefficients were equal for all the DSM, matching the post-maneuver masses (and ballistic coefficients) is intended to reduce differential drag effects.

\section{Guidance Law Derivation}

In this section, the optimal guidance problem is solved while relying on indirect dynamic optimization.

\section{A. The Hamiltonian and Co-States}

According to optimal control theory $[16,17]$, the Hamiltonian of the problem is defined as

$$
\mathcal{H}=\sum_{k=1}^{N} \mathcal{H}_{k}
$$

where

$$
\mathcal{H}_{k}=\sigma_{k} C_{k}+\gamma_{k} D_{k}+\boldsymbol{\lambda}_{\mathbf{r}_{\mathbf{k}}}{ }^{T} \mathbf{v}_{k}+\boldsymbol{\lambda}_{\mathbf{v}_{\mathbf{k}}}{ }^{T} \dot{\mathbf{v}}_{k}-\lambda_{m_{k}}\left(\sigma_{k} \frac{C_{k}}{I_{s p} g_{0}}+\gamma_{k} \frac{D_{k}}{I_{s p} g_{0}}\right)
$$

$\boldsymbol{\lambda}_{\mathbf{r}_{k}}, \boldsymbol{\lambda}_{\mathbf{v}_{k}}$, and $\lambda_{m_{k}}$ are the co-states corresponding to $\mathbf{r}_{k}, \mathbf{v}_{k}$ and $m_{k}$, respectively. 
The dynamics of the co-states are given by

$$
\begin{aligned}
\dot{\boldsymbol{\lambda}}_{r_{k}} & =-\left[\frac{\partial \mathcal{H}}{\partial \mathbf{r}_{k}}\right]^{\top} \\
\dot{\boldsymbol{\lambda}}_{v_{k}} & =-\left[\frac{\partial \mathcal{H}}{\partial \mathbf{v}_{k}}\right]^{\top} \\
\dot{\lambda}_{m_{k}} & =-\frac{\partial \mathcal{H}}{\partial m_{k}}
\end{aligned}
$$

$\mathcal{H}_{k}$ can be re-written as $\mathcal{H}_{k}=\hat{\mathcal{H}}_{k}+\overline{\mathcal{H}}_{k}$, where $\hat{\mathcal{H}}_{k}$ denotes the sum of the terms depending explicitly on the control variables $\left\{\sigma_{k}, \alpha_{k}, \delta_{k}, \gamma_{k}\right\}, k=1,2, \ldots, N$, and $\overline{\mathcal{H}}_{k}$ denotes the remaining terms. Introducing the corresponding expressions for $\dot{\mathbf{v}}_{k}$ from Eqs. (15) and defining $\boldsymbol{\lambda}_{\mathbf{v}_{k}} \triangleq\left[\lambda_{v x_{k}}, \lambda_{v y_{k}}, \lambda_{v z_{k}}\right]^{\top}$ yield the following expression:

$$
\begin{aligned}
\hat{\mathcal{H}}_{k} & =\sigma_{k}\left(C_{k}+\frac{T_{k}}{m_{k}}\left[\lambda_{v x_{k}} \cos \left(\alpha_{k}\right) \cos \left(\delta_{k}\right)+\lambda_{v y_{k}} \sin \left(\alpha_{k}\right) \cos \left(\delta_{k}\right)+\lambda_{v z_{k}} \sin \left(\delta_{k}\right)\right]-\lambda_{m_{k}} \frac{C_{k}}{I_{s p} g_{0}}\right) \\
& +\gamma_{k}\left(D_{k}-\lambda_{m_{k}} \frac{D_{k}}{I_{s p} g_{0}}\right)
\end{aligned}
$$

Recalling Eq. (23), for each $k, k=1,2, \ldots N$, Eq. (26) will be utilized to minimize the total Hamiltonian $\mathcal{H}$, with respect to $\left\{\sigma_{k}, \gamma_{k} ; \alpha_{k}, \delta_{k}\right\}$. As proven in Appendix A, the global minimum of $\hat{\mathcal{H}}_{k}$ is found either at

$$
\left\{\begin{array}{l}
\alpha_{k}^{1 *}=\arctan \left(\frac{\lambda_{v y_{k}}}{\lambda_{v x_{k}}}\right) \\
\delta_{k}^{1 *}=\arctan \left(\frac{\lambda_{v z_{k}}}{\lambda_{v x_{k}} \cos \alpha_{k}^{1 *}+\lambda_{v y_{k}} \sin \alpha_{k}^{1 *}}\right)
\end{array}\right.
$$

or at

$$
\left\{\begin{array}{l}
\alpha_{k}^{2 *}=\alpha_{k}^{1 *}+\pi \\
\delta_{k}^{2 *}=-\delta_{k}^{1 *}
\end{array}\right.
$$

The expression

$$
\tilde{\mathcal{H}}_{k} \triangleq\left[\lambda_{v x_{k}} \cos \left(\alpha_{k}\right) \cos \left(\delta_{k}\right)+\lambda_{v y_{k}} \sin \left(\alpha_{k}\right) \cos \left(\delta_{k}\right)+\lambda_{v z_{k}} \sin \left(\delta_{k}\right)\right]
$$


is computed at both solution candidates given by Eqs. (27) and (28); the candidate that yields the smallest $\tilde{\mathcal{H}}_{k}$ yields the optimal set $\left\{\alpha_{k}^{*}, \delta_{k}^{*}\right\}$. If $\lambda_{v x_{k}}=\lambda_{v y_{k}}=0$ for some $t$, then $\alpha_{k}$ is undefined and $\delta_{k}=-\operatorname{sign}\left(\lambda_{v z_{k}}\right) \pi / 2$.

Now, define the switching functions $S_{\sigma_{k}}$ as

$$
S_{\sigma_{k}} \triangleq\left(C_{k}+\frac{T_{k}}{m_{k}}\left[\lambda_{v x_{k}} \cos \left(\alpha_{k}\right) \cos \left(\delta_{k}\right)+\lambda_{v y_{k}} \sin \left(\alpha_{k}\right) \cos \left(\delta_{k}\right)+\lambda_{v z_{k}} \sin \left(\delta_{k}\right)\right]-\lambda_{m_{k}} \frac{C_{k}}{I_{s p} g_{0}}\right)
$$

The optimal value of $\sigma_{k}$ is given by

$$
\sigma_{k}^{*}=\left\{\begin{array}{l}
1, \text { if } S_{\sigma_{k}}<0 \\
0, \text { if } S_{\sigma_{k}} \geq 0
\end{array}\right.
$$

Similarly, define the switching functions $S_{\gamma_{k}}$ as

$$
S_{\gamma_{k}} \triangleq D_{k}-\lambda_{m_{k}} \frac{D_{k}}{I_{s p} g_{0}}
$$

so the optimal value of $\gamma_{k}$ is given by

$$
\gamma_{k}^{*}=\left\{\begin{array}{l}
1, \text { if } S_{\gamma_{k}}<0 \\
0, \text { if } S_{\gamma_{k}} \geq 0
\end{array}\right.
$$

Finally, $\left\{\sigma_{k}^{*}, \gamma_{k}^{*} ; \alpha_{k}^{*}, \delta_{k}^{*}\right\}$ is the argument that globally minimizes $\mathcal{H}_{k}$, and therefore the total $\mathcal{H}$. A proof of this statement is found in Appendix A.

In order to obtain the terminal constraints for the co-states, Eqs. (18)-(20) are used to 
define a function $\Phi$ as

$$
\begin{aligned}
\Phi= & +\nu_{a}\left(-\frac{\mu\left\|\mathbf{r}_{1}\left(t_{f}\right)\right\|}{\left\|\mathbf{v}_{1}\left(t_{f}\right)\right\|^{2}\left\|\mathbf{r}_{1}\left(t_{f}\right)\right\|-2 \mu}-a_{\mathcal{D}}\right) \\
& +\nu_{e}\left(\sqrt{1+\frac{\left\|\mathbf{r}_{1}\left(t_{f}\right) \times \mathbf{v}_{1}\left(t_{f}\right)\right\|^{2}}{\mu^{2}\left\|\mathbf{r}_{1}\left(t_{f}\right)\right\|}\left(\left\|\mathbf{v}_{1}\left(t_{f}\right)\right\|^{2}\left\|\mathbf{r}_{1}\left(t_{f}\right)\right\|-2 \mu\right)}-e_{\mathcal{D}}\right) \\
& +\nu_{i}\left(\arccos \left(\frac{x_{1}\left(t_{f}\right) v y_{1}\left(t_{f}\right)-y_{1}\left(t_{f}\right) v x_{1}\left(t_{f}\right)}{\left\|\mathbf{r}_{1}\left(t_{f}\right) \times \mathbf{v}_{1}\left(t_{f}\right)\right\|}\right)-i_{\mathcal{D}}\right) \\
& +\sum_{k=2}^{N}\left[\left[\nu_{1 k}, \nu_{2 k}, \nu_{3 k}\right]\left(\mathbf{r}_{k}\left(t_{f}\right)-\mathcal{R}\left(\Delta \Omega_{1 k}\right)\left[\mathbf{r}_{1}\left(t_{f}\right)+\mathbf{v}_{1}\left(t_{f}\right) \Delta t_{1 k}-\frac{\mu}{2} \frac{\mathbf{r}_{1}\left(t_{f}\right)}{\left\|\mathbf{r}_{1}\left(t_{f}\right)\right\|^{3}} \Delta t_{1 k}^{2}\right]\right)\right. \\
& +\left[\nu_{4 k}, \nu_{5 k}, \nu_{6 k}\right]\left(\mathbf{v}_{k}\left(t_{f}\right)-\mathcal{R}\left(\Delta \Omega_{1 k}\right)\left[\mathbf{v}_{1}\left(t_{f}\right)-\mu \frac{\mathbf{r}_{1}\left(t_{f}\right)}{\left\|\mathbf{r}_{1}\left(t_{f}\right)\right\|^{3}} \Delta t_{1 k}\right]\right) \\
& \left.+\nu_{7 k}\left(m_{k}\left(t_{f}\right)-m_{1}\left(t_{f}\right)\right)\right]
\end{aligned}
$$

where $\nu_{a}, \nu_{e}, \nu_{i}$, and $\nu_{l k}, l=1,2, \ldots, 7$ are all constants to be determined. Then, applying the transversality conditions, it follows that

$$
\begin{aligned}
& \boldsymbol{\lambda}_{\mathbf{r}_{k}}\left(t_{f}\right)=\left[\frac{\partial \Phi}{\partial \mathbf{r}_{k}\left(t_{f}\right)}\right]^{\top} \\
& \boldsymbol{\lambda}_{\mathbf{v}_{k}}\left(t_{f}\right)=\left[\frac{\partial \Phi}{\partial \mathbf{v}_{k}\left(t_{f}\right)}\right]^{\top} \\
& \boldsymbol{\lambda}_{m_{k}}\left(t_{f}\right)=\left[\frac{\partial \Phi}{\partial m_{k}\left(t_{f}\right)}\right]^{\top}
\end{aligned}
$$

\section{B. The Two-Point Boundary Value Problem}

Eqs. (15) and (25) constitute a set of $14 N$ ordinary differential equations, whose boundary

conditions are given by $\mathbf{y}_{k}\left(t_{i}\right)$ and Eqs. (35). Along with the set of unknowns $\nu_{a}, \nu_{e}, \nu_{i}, \nu_{m_{l}}$ ( $m=1,2, \ldots, 7$ and $k=l, 3, \ldots, N)$ and the terminal constraints Eqs. (18), Eqs. (19), and Eq. (20), this two-point boundary value problem is highly nonlinear and thus should be solved numerically.

To that end, a MATLAB ${ }^{\circledR}$ routine called bvp $4 c$, which is based on collocation methods, is 
utilized. However, due to the discontinuous behavior of $\sigma_{k}$ and $\gamma_{k}$, it is not possible to obtain a solution from the numerical solver, as it is designed for continuous dynamical systems. In order to implement the binary control laws for $\sigma_{k}$ and $\gamma_{k}$ as smooth functions, amenable for the numerical processing, Eq. (31) is replaced by

$$
\sigma_{k}=-\frac{\arctan \left(q_{\sigma} S_{\sigma_{k}}\right)+\pi / 2}{\pi}+1
$$

and Eq. (33) by

$$
\gamma_{k}=-\frac{\arctan \left(q_{\gamma} S_{\gamma_{k}}\right)+\pi / 2}{\pi}+1
$$

where $q_{\sigma}$ and $q_{\gamma}$ are parameters utilized to perform a continuation process to numerically solve the two-point boundary value problem. As $q_{\sigma} \longrightarrow \infty$, Eq. (36) tends to the corresponding binary function, in the same manner as Eq. (37) does for $q_{\gamma} \longrightarrow \infty$. The numerical solver bvp $4 \mathrm{c}$ relies on initial guesses for the whole solution. Hence, to obtain a good initial guess, it is necessary to start with low values (order of magnitude of 1 ) for $q_{\sigma}$ and $q_{\gamma}$, and then the obtained solution is used as the initial guess for the next run, where $q_{\sigma}$ and $q_{\gamma}$ are both increased by an order of magnitude. The process is iteratively continued until high enough values of $q_{\sigma}$ and $q_{\gamma}$ are reached, such that the obtained thrust profile is on-off. At orders of magnitude of $10^{4}$ for $q_{\sigma}$ and $q_{\gamma}$, the thrust profile is already perceived as an on-off profile.

\section{Closed-Loop Implementation}

The guidance law resulting from solving the two-point boundary value problem presented in Section IV.B constitutes an open-loop control law. For every set of initial states $\mathbf{Y}\left(t_{i}\right) \triangleq$ $\left[\mathbf{y}_{1}\left(t_{i}\right)^{\top}, \ldots, \mathbf{y}_{N}\left(t_{i}\right)^{\top}\right]^{\top}$ and a given terminal time $t_{f}$, the solution of the two-point boundary value problem is the optimal guidance command vector given as a function of time:

$$
\mathbf{u}\left(t ; \mathbf{Y}\left(t_{i}\right), t_{f}\right) \triangleq\left(\sigma_{k}^{*}(t), \gamma_{k}^{*}(t) ; \alpha_{k}^{*}(t), \delta_{k}^{*}(t)\right), \quad \forall t \in\left[t_{i}, t_{f}\right]
$$


In real scenarios there exist astrodynamical forces that have not been considered in the design model given in Eq. (15). Moreover, other source of errors, namely thruster misalignment, timing and magnitude, are inherent to the propulsion system. Hence, if the cluster is steered while implementing the guidance law (38), it is expected that at the end of the maneuver the actual terminal states will be different from those attained with the design model. Consequently, the constraints (19) would not be strictly satisfied. On the other hand, the constraints (20) will be satisfied as long as the thrusters are exactly fired according to $\sigma_{k}^{*}(t)$ and $\gamma_{k}^{*}(t)$, with the given design thrust level and given $I_{s p}$. The aforementioned factors may affect the long-term performance of the establishment maneuvers.

Consequently, under model uncertainty, guidance laws should be implemented in closedloop formulations. In other words, it is desired to compute the control commands as functions of the actual state rather than as functions of time, so that the guidance system is capable of coping with unmodeled perturbations.

The guidance law $\mathbf{u}\left(t ; \mathbf{Y}\left(t_{i}\right), t_{f}\right)$ derived for a given set of initial conditions $\mathbf{Y}\left(t_{i}\right)$ and a terminal time $t_{f}$ can be implemented in closed-loop by recomputing, at any time $t=\tau$, a new guidance law

$$
\mathbf{u}\left(t ; \mathbf{Y}(\tau), t_{f}-\tau\right), \quad \forall t \in\left[\tau, t_{f}\right]
$$

The frequency at which it is recomputed depends on the computational capabilities of the system.

When a maneuver is recomputed, the utilized inputs are estimates of the states. These estimates introduce errors with respect to the ideal values. In this work, every time the maneuver is recomputed, measurement errors are modeled and added to the real states, representing measured states. Hence, the state input $\hat{\mathbf{Y}}(\tau)$ used for recomputing the guidance law is given by

$$
\hat{\mathbf{Y}}(\tau)=\mathbf{Y}(\tau)+\boldsymbol{v}
$$

where $\boldsymbol{v}$ denotes the observation noise, assumed to be zero-mean multivariate Gaussian white 
noise, with covariance $R$,

$$
R=\mathrm{I}_{N} \otimes\left[\begin{array}{cc}
\operatorname{diag}\left(\sigma_{r}^{2}, \sigma_{v}^{2}\right) \otimes \mathrm{I}_{3} & \mathbf{0}_{6 \times 1} \\
\mathbf{0}_{1 \times 6} & \sigma_{m}^{2}
\end{array}\right]
$$

where $\otimes$ represents the Kronecker product.

Finally, the guidance law is recomputed as

$$
\mathbf{u}\left(t ; \hat{\mathbf{Y}}(\tau), t_{f}-\tau\right)
$$

\section{Post-Maneuver Dynamics: Selecting $\Delta t_{1 k}$ and $\Delta \Omega_{1 k}$}

After each cooperative maneuver, the evolution of $d_{i j}(t)$ can have either an increasing secular mode or a decreasing one, as can be seen in Fig. ??. Since the distances $d_{i j}\left(t_{f}\right)$ are usually rather small, a secularly increasing distance is preferable over a decreasing one, as the latter may entail a collision avoidance maneuver. The post-maneuver distance evolution depends on the parameters $\Delta t_{i j}$ and $\Delta \Omega_{i j}$ attained at the end of the maneuver. Setting these parameters properly can lead to an increasing trend as desired. The following theorem allows a better understanding of the influence of these parameters on the post-maneuver secular inter-module distance.

Theorem 1 For sufficiently small $\Delta t_{1 l}$, and for $e_{1}\left(t_{f}\right)<\sqrt{2 \sqrt{3}-3}$, the terminal states given by

$$
\begin{aligned}
& \mathbf{r}_{k}\left(t_{f}\right)=\mathcal{R}\left(\Delta \Omega_{1 k}\right)\left[\mathbf{r}_{1}\left(t_{f}\right)+\mathbf{v}_{1}\left(t_{f}\right) \Delta t_{1 k}-\frac{\mu}{2} \frac{\mathbf{r}_{1}\left(t_{f}\right)}{\left\|\mathbf{r}_{1}\left(t_{f}\right)\right\|^{3}} \Delta t_{1 k}^{2}\right], \quad \forall k=2,3, \ldots, N \\
& \mathbf{v}_{k}\left(t_{f}\right)=\mathcal{R}\left(\Delta \Omega_{1 k}\right)\left[\mathbf{v}_{1}\left(t_{f}\right)-\mu \frac{\mathbf{r}_{1}\left(t_{f}\right)}{\left\|\mathbf{r}_{1}\left(t_{f}\right)\right\|^{3}} \Delta t_{1 k}\right], \quad \forall k=2,3, \ldots, N
\end{aligned}
$$

yield $a_{k}\left(t_{f}\right)>a_{1}\left(t_{f}\right)$. 


\section{Proof}

The energy equation states

$$
\frac{v^{2}}{2}-\frac{\mu}{r}=-\frac{\mu}{2 a}
$$

where $v=\|\mathbf{v}\|$ and $r=\|\mathbf{r}\|$. The corresponding differentials satisfy

$$
v \mathrm{~d} v+\frac{\mu}{r^{2}} \mathrm{~d} r=\frac{\mu}{2 a^{2}} \mathrm{~d} a
$$

The aim is to compute $\mathrm{d} a$ for given $\mathrm{d} r$ and $\mathrm{d} v$. Since $\mu /\left(2 a^{2}\right)>0$,

$$
\operatorname{sign}(\mathrm{d} a)=\operatorname{sign}\left(v \mathrm{~d} v+\frac{\mu}{r^{2}} \mathrm{~d} r\right)
$$

Let Eq. (45) be written for $\mathrm{DSM}_{1}$ at the end of a cooperative maneuver, i.e. $v \triangleq\left\|\mathbf{v}_{1}\left(t_{f}\right)\right\|$, $r \triangleq\left\|\mathbf{r}_{1}\left(t_{f}\right)\right\|$, and $a \triangleq a_{1}\left(t_{f}\right)$. The differential values are given by $\mathrm{d} v \triangleq\left\|\mathbf{v}_{k}\left(t_{f}\right)\right\|-\left\|\mathbf{v}_{1}\left(t_{f}\right)\right\|$, $\mathrm{d} r \triangleq\left\|\mathbf{r}_{k}\left(t_{f}\right)\right\|-\left\|\mathbf{r}_{1}\left(t_{f}\right)\right\|$, and $\mathrm{d} a \triangleq a_{k}\left(t_{f}\right)-a_{1}\left(t_{f}\right)$.

From Eq. (43), an expression for $\left\|\mathbf{v}_{k}\left(t_{f}\right)\right\|$ can be written as

$$
\left\|\mathbf{v}_{k}\left(t_{f}\right)\right\|=\sqrt{v_{1}^{2}-\frac{2 \mu \Delta t_{1 k}}{r_{1}^{2}} v_{1} \cos \varsigma+\frac{\mu^{2} \Delta t_{1 k}^{2}}{r_{1}^{4}}}, \quad \forall k=2,3, \ldots, N
$$

where $\varsigma$ is the flight-direction angle, i.e. the angle between $\mathbf{r}_{1}\left(t_{f}\right)$ and $\mathbf{v}_{1}\left(t_{f}\right)$. Eq. (47) can be approximated using a second-order Taylor series expansion about $\Delta t_{1 k}=0$,

$$
\left\|\mathbf{v}_{l}\left(t_{f}\right)\right\| \simeq v_{1}-\frac{\mu \cos \varsigma}{r_{1}^{2}} \Delta t_{1 l}+\frac{\Delta t_{1 l}^{2} \mu^{2} \sin ^{2} \varsigma}{2 v_{1} r_{1}^{4}}, \quad \forall k=2,3, \ldots, N
$$

On the other hand, from Eq. (43), $\left\|\mathbf{r}_{l}\left(t_{f}\right)\right\|$ can be written as

$$
\left\|\mathbf{r}_{k}\left(t_{f}\right)\right\|=\sqrt{r_{1}^{2}+2 r_{1} v_{1} \cos \varsigma \Delta t_{1 k}-\frac{\mu}{r_{1}} \Delta t_{1 k}^{2}+v_{1}^{2} \Delta t_{1 k}^{2}-\frac{\mu v_{1} \cos \varsigma \Delta t_{1 k}^{3}}{r_{1}^{2}}+\frac{\mu^{2} \Delta t_{1 k}^{4}}{4 r_{1}^{4}}}, \quad \forall k=2,3, \ldots, N
$$


Eq. (49)) can be approximated using a second-order Taylor series expansion about $\Delta t_{1 k}=$ 0 ,

$$
\left\|\mathbf{r}_{k}\left(t_{f}\right)\right\| \simeq r_{1}+v 1 \cos \varsigma \Delta t_{1 k}-\frac{\Delta t_{1 k}^{2}}{2} \frac{\mu-r_{1} v_{1}^{2} \sin ^{2} \varsigma}{r_{1}^{2}}, \quad \forall k=2,3, \ldots, N
$$

Substituting Eqs. (48) and (50) into Eq. (45) and manipulating yields

$$
\frac{\mu}{2 a_{1}^{2}} \mathrm{~d} a_{1}=-\frac{\mu \Delta t_{1 l}^{2}}{2 r_{1}^{4}}\left(\mu \cos ^{2} \varsigma-v_{1}^{2} r_{1} \sin ^{2} \varsigma\right), \quad \forall k=2,3, \ldots, N
$$

Hence,

$$
\begin{aligned}
\operatorname{sign}\left(\mathrm{d} a_{1}\right) & =-\operatorname{sign}\left(\mu \cos ^{2} \varsigma-v_{1}^{2} r_{1} \sin ^{2} \varsigma\right) \\
& =-\operatorname{sign}\left(\mu \cos ^{2} \varsigma-\frac{h_{1}^{2}}{r_{1}}\right) \\
& =-\operatorname{sign}\left(\cos ^{2} \varsigma-\frac{p_{1}}{r_{1}}\right)
\end{aligned}
$$

where $p_{1}$ is the semi-latus rectum, $p_{1}=a_{1}\left(1-e_{1}^{2}\right)$. Defining

$$
\beta \triangleq \cos ^{2} \varsigma-\frac{p_{1}}{r_{1}}
$$

it will now be determined whether $\beta$ is positive or negative. To that end, $\beta$ is written in terms of the eccentricity $e_{1} \triangleq e_{1}\left(t_{f}\right)$ and the true anomaly $f_{1} \triangleq f_{1}\left(t_{f}\right)$. According to [18],

$$
\cos \varsigma=\frac{\mu}{h v} e \sin f
$$

where $h \triangleq\|\mathbf{h}\|=\left\|\mathbf{r}_{1}\left(t_{f}\right) \times \mathbf{v}_{1}\left(t_{f}\right)\right\|$. Substituting Eq. (54) into Eq. (53) yields

$$
\beta=\frac{\mu}{a_{1}\left(1-e_{1}^{2}\right) v_{1}^{2}} e_{1}^{2} \sin ^{2} f_{1}-1-e_{1} \cos f_{1}
$$

From Eq. (44),

$$
\frac{1}{v_{1}^{2}}=\frac{a_{1} r_{1}}{\mu\left(2 a_{1}-r_{1}\right)}
$$


Introducing Eq. (56) into Eq. (55) yields

$$
\beta=\frac{1}{\left(1+2 e_{1} \cos f_{1}+e_{1}^{2}\right)} e_{1}^{2} \sin ^{2} f_{1}-1-e_{1} \cos f_{1}
$$

Since

$$
1+2 e_{1} \cos f_{1}+e_{1}^{2} \geq 1-2 e_{1}+e_{1}^{2}=\left(1-e_{1}\right)^{2}>0
$$

$\operatorname{sgn}(\beta)=\operatorname{sgn}(\xi)$ where $\xi \triangleq \beta\left(1+2 e_{1} \cos f_{1}+e_{1}^{2}\right)$. Thus,

$$
\xi=-1-3 e_{1} \cos f_{1}-e_{1}^{2}-3 e_{1}^{2} \cos ^{2} f_{1}-e_{1}^{3} \cos f_{1}
$$

It can be proven that, for eccentricities $e_{1}<0.6812, \xi$ will always be negative. To that end, Eq. (59) is differentiated as

$$
\frac{\mathrm{d} \xi}{\mathrm{d}\left(\cos f_{1}\right)}=-3 e_{1}-6 e_{1}^{2} \cos f_{1}-e_{1}^{3}
$$

from which the maximum is obtained at

$$
\cos f_{1}=-\frac{3 e_{1}+e_{1}^{3}}{6 e_{1}^{2}}
$$

To verify that a maximum is obtained, the second derivative is computed as

$$
\frac{\mathrm{d}^{2} \xi}{\mathrm{d}\left(\cos f_{1}\right)^{2}}=-6 e_{1}^{2} \leq 0
$$

For the case in which $e_{1}=0$ it is clear that $\xi=-1<0$. The maximum of $\xi$ is

$$
\left.\xi\right|_{\cos f_{1}=-\frac{3 e_{1}+e_{1}^{3}}{6 e_{1}^{2}}}=-\frac{1}{4}+\frac{1}{2} e_{1}^{2}+\frac{1}{12} e_{1}^{4}
$$

In order for the expression on the left-hand side of Eq. (63) to be positive at its maximum, the condition for $e_{1}$ is given by $e_{1}>\sqrt{2 \sqrt{3}-3} \approx 0.68125$. In other words, if $e_{1}<0.68125$, then 
$\xi$ will always be negative, and so will $\beta$. Therefore, for $e_{1}<0.68, \operatorname{sgn}\left(\mathrm{d} a_{1}\right)=-\operatorname{sgn}(\beta)>0$.

For cases in which $e_{1}>0.68125$, one should find the anomalies $f_{1}$ at which $\xi$ switches its sign. However, the orbits of interest in this work lie in the region of low eccentricities, $e_{1}<0.1$. The reader should keep in mind that some second-order approximations were done, and these conclusions are valid in the range of $\Delta t_{1 l}$ where the Taylor expansions hold. As stated in the theorem, this result holds for DSM guided according to Eqs. (19), but it does not necessarily hold for DSM guided according to Eqs. (5). As the guidance laws developed in this work are formulated according to Eqs. (19), the stated result holds in the cases presented herein.

The implications of Theorem 1 can be understood as follows. For a sufficiently small $\Delta t_{1 k}$, Eqs. (43) yield orbital elements for $\mathrm{DSM}_{k}$ that are very similar to those of $\mathrm{DSM}_{1}$ (except the RAAN). Since $a_{1}\left(t_{f}\right)<a_{k}\left(t_{f}\right)$, according to the first-order mapping between mean and osculating elements presented in Ref. [19], one can assume that $\bar{a}_{1}\left(t_{f}\right)<\bar{a}_{k}\left(t_{f}\right)$, where $\bar{a}$ denotes the mean value of the osculating semimajor axis. Hence, the orbital period of $\mathrm{DSM}_{1}$ is smaller than that of $\mathrm{DSM}_{k}$. Therefore, if at $t=t_{f} \mathrm{DSM}_{1}$ is ahead of $\mathrm{DSM}_{k}$ in the along-track direction, $\mathrm{DSM}_{1}$ will get farther away from $\mathrm{DSM}_{k}$. Conversely, if at $t=t_{f}$, $\mathrm{DSM}_{1}$ is behind $\mathrm{DSM}_{k}$ in the along-track direction, they will get closer to each other. To analyze the influence of $\Delta t_{1 k}$ and $\Delta \Omega_{1 k}$, the following equation can be examined [3].

$$
y \approx r_{1}\left(\delta \theta+\delta \Omega \cos i_{0}\right)
$$

In Eq. (64), $y$ represents the along-track shift of $\mathrm{DSM}_{k}$ with respect to $\mathrm{DSM}_{1}$, for two DSM having very similar orbital elements. $\delta \theta$ is a slight shift in the argument of latitude, which produces a similar effect to that produced by the slight shift due to $\Delta t_{1 k}$. Moreover, $\delta \Omega$ is equivalent to the shift in RAAN $\Delta \Omega_{1 k}$, while $i_{0}$ represent the inclination of $\mathrm{DSM}_{1}$.

Assume that for given $\Delta t_{1 k_{1}}$ and $\Delta \Omega_{1 k_{1}}$, the secular component of $d_{1 k}$ is decreasing. $\mathrm{DSM}_{k}$ is represented by the black circle in Fig. 4. For the same $\mathbf{r}_{1}\left(t_{f}\right)$ and $\mathbf{v}_{1}\left(t_{f}\right)$, reducing 


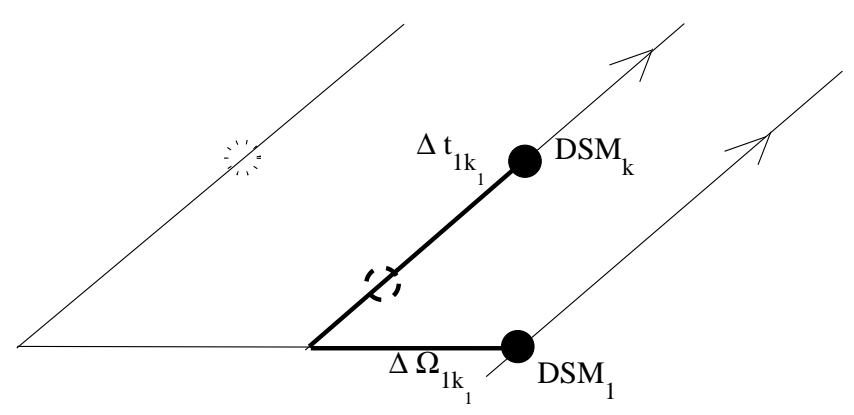

Figure 4. Influence of $\Delta t_{1 k}$ and $\Delta \Omega_{1 k}$ in shifting the modules in the along-track direction.

to some $\Delta t_{1 k_{2}}<\Delta t_{1 k_{1}}$ (dashed circle in Fig. 4) moves $\mathrm{DSM}_{k}$ backward in the along-track direction, while still $\bar{a}_{1}\left(t_{f}\right)<\bar{a}_{k}\left(t_{f}\right)$. Hence, it induces a secular distance growth. On the other hand, for the same $\mathbf{r}_{1}\left(t_{f}\right)$ and $\mathbf{v}_{1}\left(t_{f}\right)$, keeping the same $\Delta t_{1 k_{1}}$ and reducing $\Delta \Omega_{1 k}$ (dotted circle in Fig. 4) moves $\mathrm{DSM}_{k}$ backward in the along-track direction for inclinations $0^{\circ}<i<90^{\circ}$, contributing to a growing secular distance. Conversely, enlarging $\Delta \Omega_{1 k}$ moves $\mathrm{DSM}_{k}$ forward in the along-track direction for inclinations $90^{\circ}<i<180^{\circ}$, and thus causing decreasing distance evolution.

In order to illustrate these effects, an example of two modules is provided. For $\Delta \Omega_{1 k}=$ $0.05^{\circ}$ and $\Delta t_{1 k}=1 \mathrm{sec}$, the behavior seen in Fig. 5(a) is obtained. Keeping $\Delta \Omega_{1 k}=0.05^{\circ}$ but reducing to $\Delta t_{1 k}=0.1 \mathrm{sec}$, yields the secular growth seen in Fig. 5(b).

With respect to the secular component of the distance between $\mathrm{DSM}_{k}$ and $\mathrm{DSM}_{j}$, a similar argument can be used. According to Eq. (51), it is seen that the larger $\Delta t$, the higher $\mathrm{d} a_{1}$, and thus the higher the semimajor axis of $\mathrm{DSM}_{k}$. If $\Delta t_{1 k} \gtrless \Delta t_{1 j}, a_{k}\left(t_{f}\right) \gtrless a_{j}\left(t_{f}\right)$, and thus $\bar{a}_{k}\left(t_{f}\right) \gtrless \bar{a}_{j}\left(t_{f}\right)$. Therefore, for given $\Delta t_{1 k}$ and $\Delta t_{1 j}$, one can exploit $\Delta \Omega_{1 k}$ and $\Delta \Omega_{1 j}$ to induce a growing secular behavior of the distance between $\mathrm{DSM}_{k}$ and $\mathrm{DSM}_{j}$. 


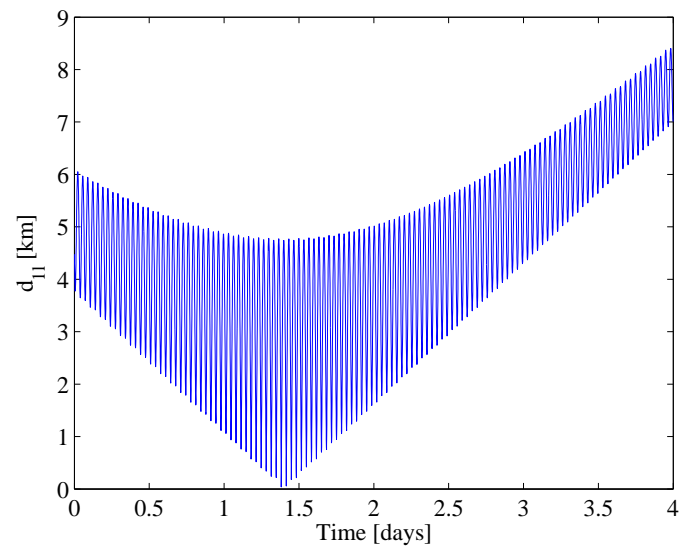

(a) Post-maneuver decreasing secular distance.

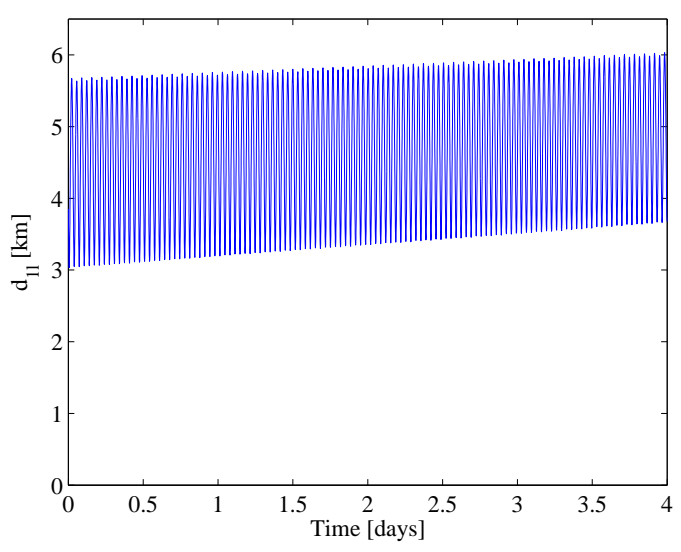

(b) Post-maneuver increasing secular distance.

Figure 5. Post-maneuver secular behavior.

\section{Simulations}

This section provides simulation results showing how the optimal cooperative guidance laws steer the satellites to achieve the required constraints on the terminal states, while minimizing the fuel consumption. Furthermore, it is illustrated how these constraints generate slowly-drifting relative distances among the modules while forcing $\mathrm{DSM}_{1}$ to track a desired reference orbit.

After the deployment in orbit by the launch vehicle, the modules perform a cooperative establishment maneuver, based on the design model described by Eqs. (15), to reach a set of states such that Eqs. (19) and (20) hold, and a subset of the osculating elements of $\mathrm{DSM}_{1}$ (i.e., $\left.a_{1}, e_{1}, i_{1}\right)$ attain the desired values, according to Eq. (18). Once these specific states are reached within a prescribed time interval, all the modules fly ballistically until any of the constraints

$$
D_{\min } \leq d_{12}(t) \leq D_{\max }, \quad D_{\min } \leq d_{23}(t) \leq D_{\max }, \quad D_{\min } \leq d_{31}(t) \leq D_{\max }
$$

or the constraints (2) is violated.

According to Section II, if one of the constraints (65) or (??) is exceeded, a re-establishment maneuver will be performed to reconfigure the cluster and then it will be allowed to fly bal- 
listically again. This procedure would be applied throughout the mission lifetime. The dynamical model used to propagate the ballistic arcs - from now on named the coasting model - includes a $21 \times 21$ EGM96 gravitational model, drag according to the ISA-1976 model, solar radiation pressure with a dual-cone shadow model, luni-solar attraction, tides and relativistic effects.

Largely based on the SAMSON project [20], the following applicative scenario is simulated, considering a cluster of three DSM.

\section{A. Test Case}

The bounds for the relative distances are set as $D_{\min }=0.1 \mathrm{~km}$ and $D_{\max }=250 \mathrm{~km}$. The time frame for a complete cooperative maneuver was chosen as $t_{f}=5800 \mathrm{sec}$. The mission lifetime is assumed to be 3 years. The initial conditions of the DSM, after launcher release, as well as the required final orbital elements for $\mathrm{DSM}_{1}$ are listed in Table 2. The initial states represent a typical launch vehicle dispersion around the nominal injection orbit (rows 1,2 and 3 of Table 2), while the desired values of $a_{\mathcal{D}}, e_{\mathcal{D}}, i_{\mathcal{D}}$ correspond to the orbital elements for $\mathrm{DSM}_{1}$ at $t=t_{f}$ (row 4 of Table 2 ). Furthermore, the following parameters are assumed: $T_{n}=0.015 \mathrm{~N}, I_{s p}=70 \mathrm{~s}, C_{D_{1}}=C_{D_{2}}=C_{D_{3}}=2.2, S_{1}=S_{2}=S_{3}=0.11 \mathrm{~m}^{2}$, density during the maneuver time frame $\rho=1.13710^{-13} \mathrm{~kg} / \mathrm{m}^{3}, \Delta t_{12}=0.1 \mathrm{~s}, \Delta \Omega_{12}=-0.05^{\circ}$, $\Delta t_{13}=0.05 \mathrm{~s}, \Delta \Omega_{13}=-0.025^{\circ}$. The tolerances for the orbital elements of $\mathrm{DSM}_{1}$ are given as $\left|a-a_{\mathcal{D}}\right|=20 \mathrm{~km},\left|e-e_{\mathcal{D}}\right|=0.0025$, and $\left|i-i_{\mathcal{D}}\right|=0.25^{\circ}$.

Using the boundary conditions of Table 2, the three modules first perform a cooperative cluster establishment maneuver (see Fig. 6). During the maneuver, the simulated dynamical model includes perturbations of the two-body force field, given by the $J_{2}$ term of the zonal potential and drag with a constant density $\rho$, according to Eqs. (15). The constraints forcing $\mathrm{DSM}_{1}$ to track a reference orbit (Eqs. (18)), as well as the relative displacement constraint (Eqs. (19)) and the mass constraint (Eqs. (20)) are all active.

Fig. 6(a), from top to bottom, presents an on-off control profile for the three modules (orbit control, $\left.\sigma_{k}\right)$ and shows that the intentional mass disposal $\left(\gamma_{k}\right)$ is required in this mission 
Table 2. Sample mission specifications. Initial orbital elements for $\mathrm{DSM}_{1}$ (row 1), $\mathrm{DSM}_{2}$ (row 2) $\mathrm{DSM}_{3}$ (row 3), as well as final (desired) states for $\mathrm{DSM}_{1}$ (row 4).

\begin{tabular}{cccccccc}
$\mathrm{DSM}$ & $a[\mathrm{~km}]$ & $e[-]$ & $i[\mathrm{deg}]$ & $\Omega[\mathrm{deg}]$ & $\omega[\mathrm{deg}]$ & $f[\mathrm{deg}]$ & $m[\mathrm{~kg}]$ \\
\hline $\mathrm{DSM}_{1}\left(t=t_{i}\right)$ & 6982 & 0.0035 & 51.5334 & 0.01667 & 10.01 & 50.1 & 6 \\
$\mathrm{DSM}_{2}\left(t=t_{i}\right)$ & 6977 & 0.0025 & 51.4667 & -0.01667 & 9.99 & 49.9 & 6 \\
$\mathrm{DSM}_{3}\left(t=t_{i}\right)$ & 6979.25 & 0.002375 & 51.5417 & 0.02083 & 9.9875 & 49.875 & 6 \\
$\mathrm{DSM}_{1}\left(t=t_{f}\right)$ & 6978 & 0.003 & 51.5 & - & - & - & -
\end{tabular}
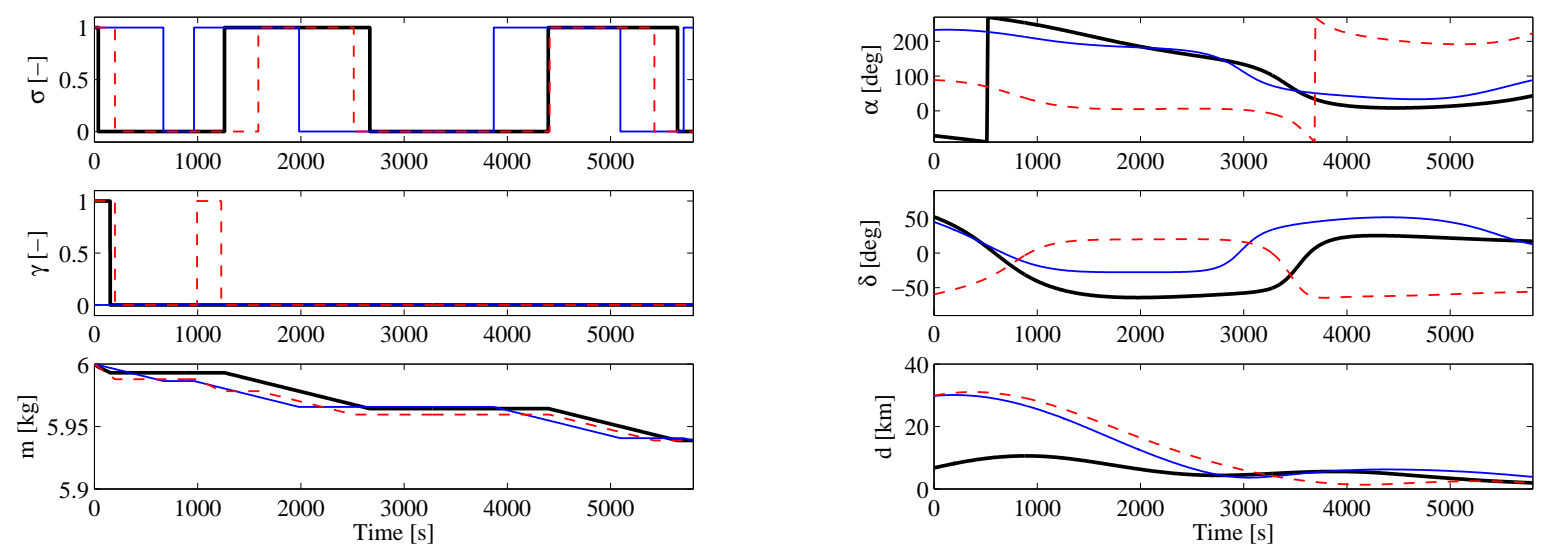

(a) Control variables $\sigma, \gamma$ and mass of $\mathrm{DSM}_{1}, \mathrm{DSM}_{2}$ and $\mathrm{DSM}_{3}$.

(b) Control variables $\alpha, \delta$ and inter-module distance between $\mathrm{DSM}_{1}, \mathrm{DSM}_{2}$ and $\mathrm{DSM}_{3}$.
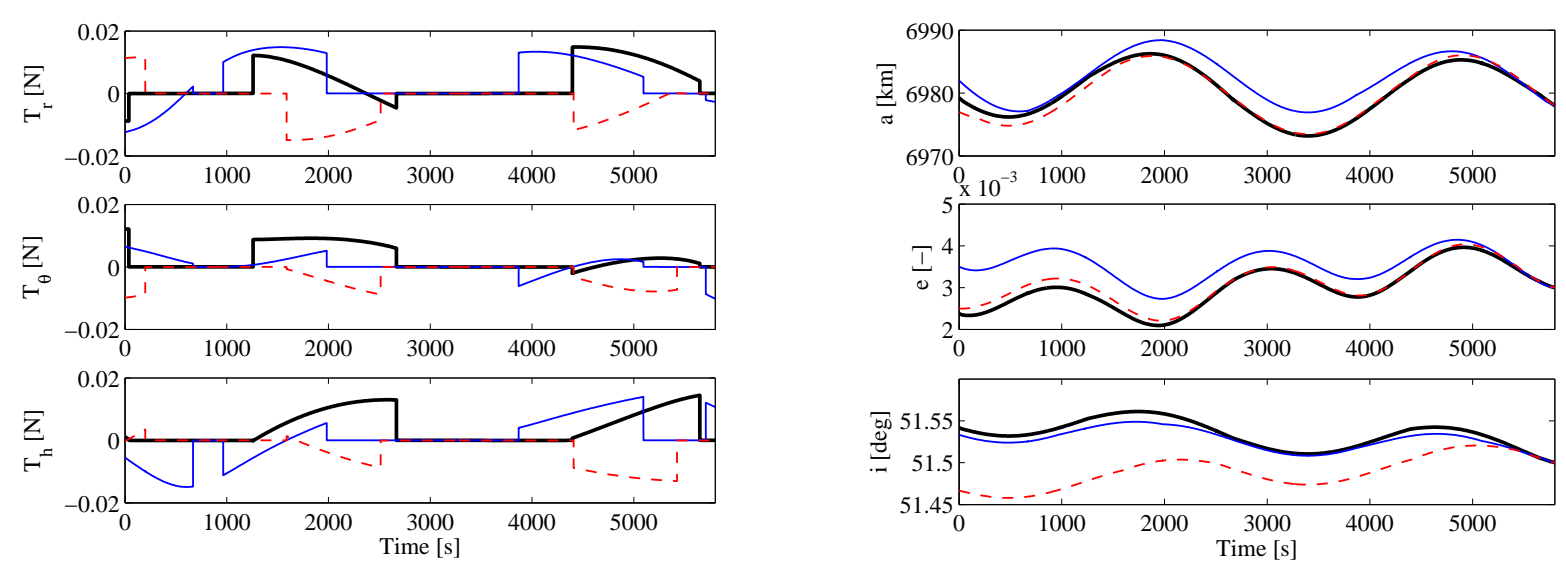

(c) Control variables $T_{r}, T_{\theta}$ and $T_{h}$ of $\mathrm{DSM}_{1}, \mathrm{DSM}_{2}$ and $\mathrm{DSM}_{3}$.

(d) Tracked orbital elements for $\mathrm{DSM}_{1}, \mathrm{DSM}_{2}$ and $\mathrm{DSM}_{3}$.

Figure 6. Cluster establishment after orbit injection. Solid line: $\mathrm{DSM}_{1}$, dashed line: $\mathrm{DSM}_{2}$, bold line: $\mathrm{DSM}_{3}$. 


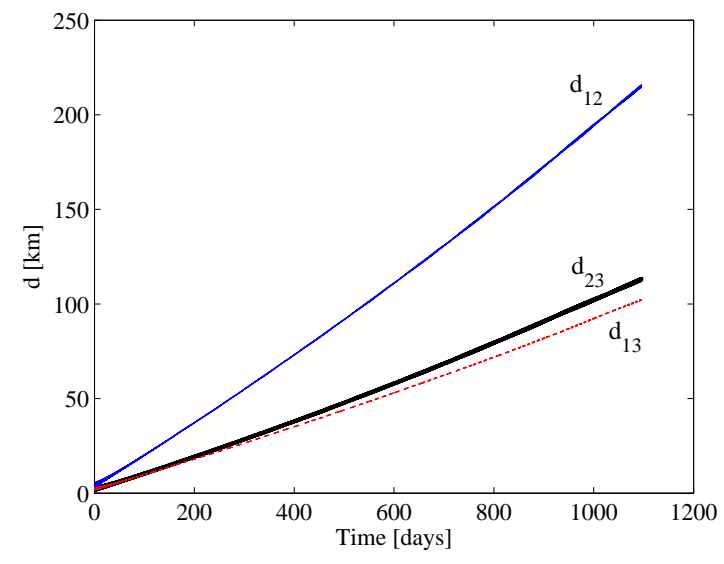

(a) Inter-module distance between $\mathrm{DSM}_{1}$ and $\mathrm{DSM}_{2}, \mathrm{DSM}_{1}$ and $\mathrm{DSM}_{3}$, and $\mathrm{DSM}_{2}$ and $\mathrm{DSM}_{3}$.
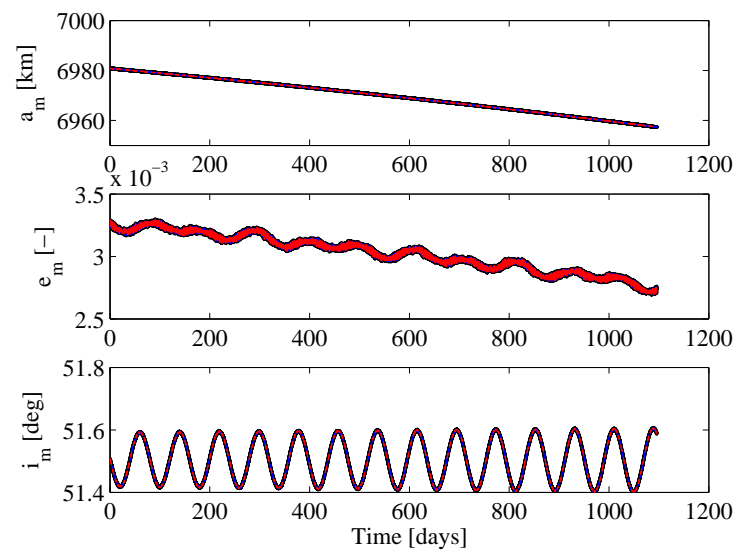

(b) Tracked orbital elements of $\mathrm{DSM}_{1}, \mathrm{DSM}_{2} \mathrm{DSM}_{3}$.

Figure 7. Cluster performance for the entire mission lifetime, considering a $21 \times 21$ EGM96 gravitational field, drag according to the ISA-1976 model, solar radiation pressure with dual-cone shadow, luni-solar attraction, tides and relativistic effects.

scenario to let all the modules attain the same value of final mass. Fig. 6(b) depicts the thrust pointing angles, $\alpha_{k}$ and $\delta_{k}$. Moreover, it is seen that the inter-module distance is kept bounded during the establishment maneuver (Fig. 6(b), bottom). In Fig. 6(c) the exerted thrust vector is resolved into radial, along-track, and cross-track components. Fig. 6(d) shows the time history of the semimajor axes, eccentricities and inclinations during the maneuver.

Assuming that the maneuver is perfectly performed, i.e. according to the nominal solution, the DSM are then allowed to coast under the natural forces acting on them (see Fig. 7), simulated by the coasting model, until any violation of the constraints described by Eqs. (1) and Eqs. (2) occurs.

Establishing the proper relative position among the modules and achieving the same ballistic coefficient renders the inter-module distance slowly drifting, but within the prespecified bounds for the entire mission lifetime (see Fig. 7(a)). Moreover, the limits on the orbital elements of $\mathrm{DSM}_{1}$ (which tracks the reference path) are never exceeded (see Fig. 7(b)), allowing the cluster to accomplish the mission requirements with only one initial establishment maneuver, consuming approximately 60 grams of propellant per module. 


\section{B. Closed-Loop Implementation of the Guidance Law}

This section illustrates closed-loop implementation of the derived guidance law, according to Eq. (42), and compares the performance of closed-loop implementation to an open-loop execution of the same maneuver.

To that end, a maneuver lasting 5800 seconds is determined for a cluster composed of $N=3$ DSM. To compute the maneuver, the initial conditions listed in Table 2 are considered. As opposed to the case elaborated in Section A, there are no specific requirements on the payload orbit, and therefore no constraints were imposed on the semimajor axis, eccentricity and inclination of $\mathrm{DSM}_{1}$. Moreover, the initial masses of the three modules are set as as $m_{k}\left(t_{i}\right)=8 \mathrm{~kg}$. The following parameters were assumed: $T_{n}=0.080 \mathrm{~N}, I_{s p}=60 \mathrm{~s}$, $C_{D_{1}}=C_{D_{2}}=C_{D_{3}}=2.2, S_{1}=S_{2}=S_{3}=0.11 \mathrm{~m}^{2}, \Delta t_{12}=0.1 \mathrm{~s}, \Delta \Omega_{12}=-0.05^{\circ}$ and $\Delta t_{13}=0.05 \mathrm{~s}, \Delta \Omega_{13}=-0.025^{\circ}$. For the design model, the constant density is taken as $\rho=1.13710^{-13} \mathrm{~kg} / \mathrm{m}^{3}$.

In order to simulate the closed-loop implementation, the two-point boundary value problem is solved every 1160 seconds during the maneuver ${ }^{1}$. For recomputing the solution given by Eq. (42), erroneous measurements are generated according to Eq. (40). The entries of the matrix $R$, stated in Eq. (41), are given as $\sigma_{r}=5 \mathrm{~m}, \sigma_{v}=0.02 \mathrm{~m} / \mathrm{s}$, and $\sigma_{m}=5 \cdot 10^{-5} \mathrm{~kg}$. Moreover, the actual execution of the maneuver was simulated according to the obtained solution (42) but while considering a more complete astrodynamical model including a $10 \times 10$ geopotential, density according to the model NRLMSISE-00, and errors in the magnitude of the implemented thrust. The errors in the thrust magnitude were modeled as

$$
T_{k}(t)=T_{k}^{n}(t)+\epsilon_{T_{k}}
$$

where $T_{k}^{n}(t)$ denotes the nominal thrust $T_{n} \sigma_{k}(t)$ as obtained from the solution (42), and $\epsilon_{T_{k}} \sim \mathcal{N}(0,0.010 \mathrm{~N})$. Notice that $\epsilon_{T_{k}}$ was held constant along the maneuver.

In order to quantify the comparison between open-loop and closed-loop implementation,

\footnotetext{
${ }^{1}$ It would be desirable to recompute the maneuver at a higher frequency, but due to the required computational burden it is done every 1160 seconds.
} 
the performance of the maneuver is measured by means of three metrics defined as:

$$
\begin{aligned}
\Delta_{\mathbf{r}_{1 k}} & =\left\|\mathbf{r}_{k}\left(t_{f}\right)-\mathcal{R}\left(\Delta \Omega_{1 k}\right)\left[\mathbf{r}_{1}\left(t_{f}\right)+\mathbf{v}_{1}\left(t_{f}\right) \Delta t_{1 l}-\frac{\mu}{2} \frac{\mathbf{r}_{1}\left(t_{f}\right)}{\left\|\mathbf{r}_{1}\left(t_{f}\right)\right\|^{3}} \Delta t_{1 k}^{2}\right]\right\| \quad k=2,3, \ldots, N \\
\Delta_{\mathbf{v}_{1 k}} & =\left\|\mathbf{v}_{k}\left(t_{f}\right)-\mathcal{R}\left(\Delta \Omega_{1 l}\right)\left[\mathbf{v}_{1}\left(t_{f}\right)-\mu \frac{\mathbf{r}_{1}\left(t_{f}\right)}{\left\|\mathbf{r}_{1}\left(t_{f}\right)\right\|^{3}} \Delta t_{1 k}\right]\right\| \quad k=2,3, \ldots, N \\
\Delta_{m_{1 k}} & =\left|m_{k}\left(t_{f}\right)-m_{1}\left(t_{f}\right)\right|
\end{aligned}
$$

$\Delta_{\mathbf{r}_{1 k}}, \Delta_{\mathbf{v}_{1 k}}$, and $\Delta_{m_{1 k}}$ measure the violation of constraints (19) and (20). If $\Delta_{\mathbf{r}_{1 k}}=\Delta_{\mathbf{v}_{1 k}}=$ $\Delta_{m_{1 k}}=0$, then a perfect maneuver was performed as the terminal constraints were perfectly achieved. However, due to measurement and actuation errors, as well as modelling errors, these metrics attain positive values. In this sense, the smaller the achieved metric values, the better the performance of the maneuver. If the attained metrics are sufficiently small, it is expected that the inter-module distances would have a relatively low drift.

In order to perform the comparison, both closed-loop and open-loop executions of the same maneuver were corrupted by the same level of uncertainties in the measurements, and the same level of uncertainty in the thrust magnitudes of each DSM. The same astrodynamical model is used for the open-loop and closed-loop guidance. A total of 22 Monte-Carlo simulations of closed-loop and open-loop maneuvers were performed, where the random parameters are $\epsilon_{T_{k}}$ (corresponding to the thrust magnitude of each DSM) and $\boldsymbol{v}$ (representing the noise applied to the measurements). The obtained metrics (67), for all the cases, are exhibited as histograms in Figure 8.

In the histograms, the light bars refer to the open-loop execution, and the dark bars represent the closed-loop implementation of the guidance law. Fig. 8 shows that the closedloop implementation reduces the obtained errors in attaining the desired terminal constraints (19) and (20).

Due to actuation errors related to the thrust magnitude, the obtained final masses (and ballistic coefficients) are not equal, i.e. (19) is not perfectly achieved. Moreover, in these examples, the mass disposal throttle parameter satisfies $\gamma_{k}(t)=0$. This result stems from the 

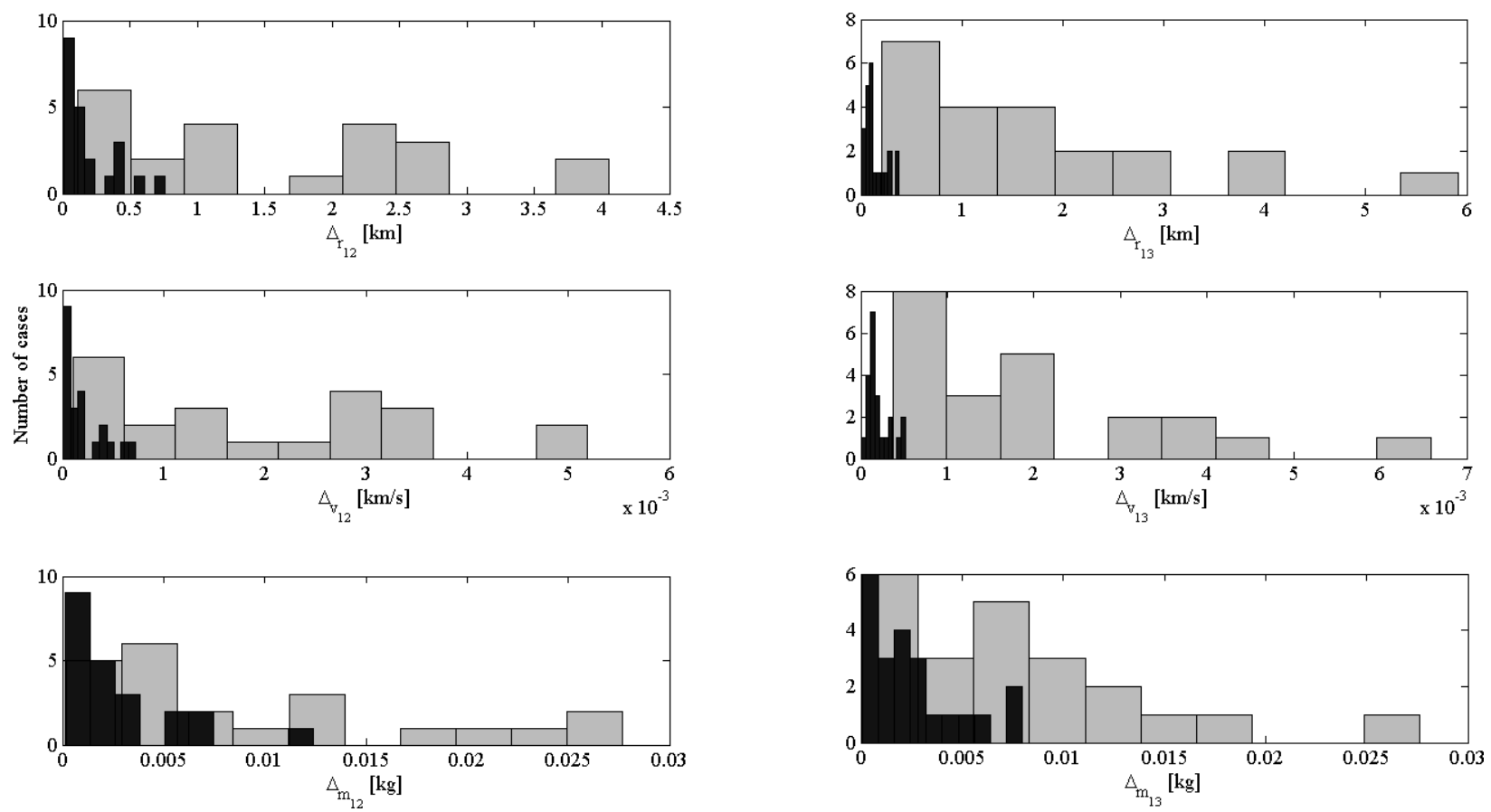

Figure 8. Histograms of the obtained values for the metrics $\Delta_{\mathbf{r}_{1 k}}, \Delta_{\mathbf{v}_{1 k}}$, and $\Delta_{m_{1 k}}$. A comparison of open-loop (light bars) and closed-loop (dark bars) implementation.

fact that in these examples no absolute orbit is targeted, and there are no large differences in the initial masses of each closed-loop solution. Therefore, the optimizer finds trajectories for the three DSM that attain the terminal constraints (19) and (20), without the need to trigger the intentional mass disposal.

\section{Conclusions}

Keeping a cluster of modules within bounded distances for prolonged intervals of time is challenging due to differential perturbation effects. However, cooperative guidance can mitigate the distance drift. To achieve that, distance-keeping constraints should be targeted. It is important to design cooperative maneuvers that minimize the differences in ballistic coefficients. This effect is significant for low Earth orbit clusters due to differential drag effects. If an optimal guidance law as the one developed herein is successful in reaching the desired terminal states, then a very low relative drift will be obtained for long-term missions. However, in a real implementation, due to measurement and actuation errors, 
open-loop maneuvers might steer the system to terminal states that are far from satisfying the aforementioned constraints. In this case, a closed-loop scheme such as the one proposed herein should be implemented. The closed-loop scheme is much less sensitive to thrust and measurement errors.

\section{Acknowledgments}

This work was supported by the European Research Council Starting Independent Researcher Grant - 278231: Flight Algorithms for Disaggregated Space Architectures (FADER).

\section{Appendix A: Minimization of $\hat{\mathcal{H}}_{k}$}

This section proves that the global minimum of $\hat{\mathcal{H}}_{k}$, defined by Eq. (26), is given by Eqs. (27)-(28). Recall that $C_{k}, T_{k}, I_{s p}$ and $g_{0}$ are given positive constants, whereas $\lambda_{v x_{k}}$, $\lambda_{v y_{k}}, \lambda_{v z_{k}}$, and $\lambda_{m_{k}}$ are time-varying Lagrange coefficients. Moreover, for the optimization problem, $\sigma_{k} \in[0,1]$ and $\gamma_{k} \in[0,1]$. $\hat{\mathcal{H}}_{k}$, as a function of $\alpha_{k}$ and $\delta_{k}$, is continuous and smooth. If, for a given time $t, \lambda_{v x_{k}}=\lambda_{v y_{k}}=0$ and $\lambda_{v y_{z}} \neq 0$, then $\delta_{k}^{*}=-\operatorname{sgn}\left(\lambda_{v z_{k}}\right) \pi / 2$ minimizes $\hat{\mathcal{H}}_{k}$, which yields $\cos \left(\delta_{k}\right)=0$, and $\alpha_{k}$ is undefined. If, for a given time $t, \lambda_{v x_{k}}=\lambda_{v y_{k}}=\lambda_{v z_{k}}=0$ then it is a singular point and both $\alpha_{k}$ and $\delta_{k}$ are undefined, i.e. any $\alpha_{k}$ and $\delta_{k}$ yield the same value of $\hat{\mathcal{H}}_{k}$. For any other case, the following partial derivative is nullified:

$$
\frac{\partial \hat{\mathcal{H}}_{k}}{\partial \alpha_{k}}=\sigma_{k} \frac{T_{k}}{m_{k}}\left[-\lambda_{v x_{k}} \sin \left(\alpha_{k}\right) \cos \left(\delta_{k}\right)+\lambda_{v y_{k}} \cos \left(\alpha_{k}\right) \cos \left(\delta_{k}\right)\right]=0
$$

Since $\cos \left(\delta_{k}\right) \neq 0$

$$
\alpha_{k}^{*}=\left\{\begin{array}{l}
\alpha_{k}^{1 *} \triangleq \arctan \left(\frac{\lambda_{v y_{k}}}{\lambda_{v x_{k}}}\right) \\
\alpha_{k}^{2 *} \triangleq \arctan \left(\frac{\lambda_{v y_{k}}}{\lambda_{v x_{k}}}\right)+\pi
\end{array}\right.
$$


Now, the partial derivative with respect to $\delta_{k}$ is nullified:

$$
\frac{\partial \hat{\mathcal{H}}_{k}}{\partial \delta_{k}}=\sigma_{k} \frac{T_{k}}{m_{k}}\left[-\lambda_{v x_{k}} \cos \left(\alpha_{k}\right) \sin \left(\delta_{k}\right)-\lambda_{v y_{k}} \sin \left(\alpha_{k}\right) \sin \left(\delta_{k}\right)+\lambda_{v z_{k}} \cos \left(\delta_{k}\right)\right]=0
$$

from which

$$
\delta_{k}^{*}=\left\{\begin{array}{l}
\delta_{k}^{11 *} \triangleq \arctan \left(\frac{\lambda_{v z_{k}}}{\lambda_{v x_{k}} \cos \left(\alpha_{k}^{1 *}\right)+\lambda_{v y_{k}} \sin \left(\alpha_{k}^{1 *}\right)}\right) \\
\delta_{k}^{21 *} \triangleq \arctan \left(\frac{\lambda_{v z_{k}}}{\lambda_{v x_{k}} \cos \left(\alpha_{k}^{2 *}\right)+\lambda_{v y_{k}} \sin \left(\alpha_{k}^{2 *}\right)}\right)=-\delta_{k}^{11 *} \\
\delta_{k}^{12 *} \triangleq \arctan \left(\frac{\lambda_{v z_{k}}}{\lambda_{v x_{k}} \cos \left(\alpha_{k}^{1 *}\right)+\lambda_{v y_{k}} \sin \left(\alpha_{k}^{1 *}\right)}\right)+\pi=\delta_{k}^{11 *}+\pi \\
\delta_{k}^{22 *} \triangleq \arctan \left(\frac{\lambda_{v z_{k}}}{\lambda_{v x_{k}} \cos \left(\alpha_{k}^{2 *}\right)+\lambda_{v y_{k}} \sin \left(\alpha_{k}^{2 *}\right)}\right)+\pi=-\delta_{k}^{11 *}+\pi
\end{array}\right.
$$

From Eqs. (69) and (71), the local minima or maxima of $\hat{\mathcal{H}}_{k}$ are found at $\left(\alpha_{k}^{1 *}, \delta_{k}^{11 *}\right)$ or $\left(\alpha_{k}^{2 *}, \delta_{k}^{21 *}\right)$ or $\left(\alpha_{k}^{1 *}, \delta_{k}^{12 *}\right)$ or $\left(\alpha_{k}^{2 *}, \delta_{k}^{22 *}\right)$. Recall the definition of $\tilde{\mathcal{H}}_{k}$ according to Eq. (29). By direct computation it can be seen that

$$
\begin{aligned}
& \left.\tilde{\mathcal{H}}_{k}\right|_{\left(\alpha_{k}^{1 *}, \delta_{k}^{12 *}\right)}=-\left.\tilde{\mathcal{H}}_{k}\right|_{\left(\alpha_{k}^{1 *}, \delta_{k}^{11 *}\right)} \\
& \left.\tilde{\mathcal{H}}_{k}\right|_{\left(\alpha_{k}^{2 *}, \delta_{k}^{21 *}\right)}=-\left.\tilde{\mathcal{H}}_{k}\right|_{\left(\alpha_{k}^{1 *}, \delta_{k}^{11 *}\right)} \\
& \left.\tilde{\mathcal{H}}_{k}\right|_{\left(\alpha_{k}^{2 *}, \delta_{k}^{22 *}\right)}=\left.\tilde{\mathcal{H}}_{k}\right|_{\left(\alpha_{k}^{1 *}, \delta_{k}^{11 *}\right)}
\end{aligned}
$$

To find the global minimum it is sufficient to evaluate $\left.\tilde{\mathcal{H}}_{k}\right|_{\left(\alpha_{k}^{1 *}, \delta_{k}^{11 *}\right)}$ and $\left.\tilde{\mathcal{H}}_{k}\right|_{\left(\alpha_{k}^{2 *}, \delta_{k}^{21 *}\right)}$, and determine which is the negative one, which constitutes the global minimum. The argument that minimize $\tilde{\mathcal{H}}_{k}$ is selected as either $\left(\alpha_{k}^{1 *}, \delta_{k}^{11 *}\right)$ or $\left(\alpha_{k}^{2 *}, \delta_{k}^{21 *}\right)$ such that $\tilde{\mathcal{H}}_{k}$ is negative. In this manner, $\alpha_{k}^{*} \in[-\pi / 2,3 \pi / 2) \operatorname{rad}$ and $\delta_{k}^{*} \in[-\pi / 2, \pi / 2] \operatorname{rad}$.

Since $\sigma_{k}$ is nonnegative, to determine the argument $\sigma_{k}$ that minimize $\hat{\mathcal{H}}_{k}$ it is necessary 
to evaluate the minimum of the expression multiplying $\sigma_{k}$, i.e.

$$
S_{\sigma_{k}} \triangleq C_{k}+\frac{T_{k}}{m_{k}}\left[\lambda_{v x_{k}} \cos \left(\alpha_{k}^{*}\right) \cos \left(\delta_{k}^{*}\right)+\lambda_{v y_{k}} \sin \left(\alpha_{k}^{*}\right) \cos \left(\delta_{k}^{*}\right)+\lambda_{v z_{k}} \sin \left(\delta_{k}^{*}\right)\right]-\lambda_{m_{k}} \frac{C_{k}}{I_{s p} g_{0}}
$$

Then

$$
\sigma_{k}^{*}=\left\{\begin{array}{l}
1, \text { if } S_{\sigma_{k}}<0 \\
0, \text { if } S_{\sigma_{k}} \geq 0
\end{array}\right.
$$

Since $\gamma_{k}$ is nonnegative, in order to determine the argument $\gamma_{k}$ that minimize $\hat{\mathcal{H}}_{k}$ it is necessary to evaluate the minimum of the expression multiplying $\gamma_{k}$, i.e.

$$
S_{\gamma_{k}} \triangleq D_{k}-\lambda_{m_{k}} \frac{D_{k}}{I_{s p} g_{0}}
$$

Then $\gamma_{k}$ is given by

$$
\gamma_{k}^{*}=\left\{\begin{array}{l}
1, \text { if } S_{\gamma_{k}}<0 \\
0, \text { if } S_{\gamma_{k}} \geq 0
\end{array}\right.
$$

\section{References}

${ }^{1}$ Brown, O. and Eremenko, P., "Fractionated Space Architectures: A Vision for Responsive Space," 4th Responsive Space Conference, 4th Responsive Space Conference, Los Angeles, CA, 2006.

${ }^{2}$ Mathieu, C. and Weigel, L., "Assessing the Fractionated Spacecraft Concept," AIAA Space 2006 Conference and Exposition, AIAA-2006-7212, San Jose, CA, 2006.

${ }^{3}$ Alfriend, K., Vadali, S., Gurfil, P., How, J., and Breger, L., Spacecraft Formation Flying: Dynamics,Control and Navigation, Elseiver, Oxford, UK, 2010, Chapter 2, pp. 24, 35-36, and Chapter 7, pp. 146.

${ }^{4}$ Vadali, S., Schaub, H., and Alfriend, K., "Initial Conditions and Fuel-optimal Control for Formation Flying of Satellites," AIAA GNC Conference, Portland, OR, 1999, Paper No. AIAA 99-4265.

${ }^{5}$ Schaub, H. S. and Alfriend, K. T., " $J_{2}$ Invariant Relative Orbits for Spacecraft Formations," Celestial Mechanics and Dynamical Astronomy, Vol. 79, No. 2, February 2001, pp. 77-95.

${ }^{6}$ Mishne, D., "Formation Control of Satellites Subject to Drag Variations and $J_{2}$ Perturbations," Journal of Guidance, Control and Dynamics, Vol. 27, No. 4, July-August 2004, pp. 685-692. 
${ }^{7}$ Beigelman, I. and Gurfil, P., "Optimal Fuel-Balanced Impulsive Formationkeeping for Perturbed Spacecraft Orbits," Journal of Guidance, Control and Dynamics, Vol. 31, No. 5, September-October 2008, pp. $1266-1283$.

${ }^{8}$ Martinusi, V. and Gurfil, P., "Solutions and Periodicity of Satellite Relative Motion Under Even Zonal Harmonics Perturbations," Celestial Mechanics and Dynamical Astronomy, Vol. 111, No. 4, 2011, pp. $387-414$.

${ }^{9} \mathrm{Xu}, \mathrm{M}$., Wang, Y., and $\mathrm{Xu}$, S., "On the existence of $J_{2}$ invariant relative orbits from the dynamical system point of view," Celestial Mechanics and Dynamical Astronomy, Vol. 112, 2012, pp. $427-444$.

${ }^{10}$ Mazal, L. and Gurfil, P., "Cluster Flight Algorithms for Disaggregated Satellites," Journal of Guidance, Control and Dynamics, Vol. 36, No. 1, January-February 2013, pp. 124-135.

${ }^{11}$ De Queiroz, M., Kapila, V., and Yan, Q., "Adaptive Nonlinear Control of Multiple Spacecraft Formation Flying," Journal of Guidance, Control, and Dynamics, Vol. 23, No. 3, 2000, pp. 385-390.

${ }^{12}$ Wang, Z., Khorrami, F., and Grossman, W., "Robust Adaptive Control of Formationkeeping for a Pair of Satellites," Proceedings of the American Control Conference, 2000, Vol. 2, IEEE, 2000, pp. 834-838.

${ }^{13}$ Izzo, D. and Pettazzi, L., "Autonomous and Distributed Motion Planning for Satellite Swarm," Journal of Guidance Control and Dynamics, Vol. 30, No. 2, 2007, pp. 449-459.

${ }^{14}$ Gergaud, J., Haberkorn, T., et al., "Homotopy Method for Minimum Consumption Orbit Transfer Problem," ESAIM Controle Optimisation et Calcul des Variations, Vol. 12, 2006, pp. 294.

${ }^{15}$ Guo, T., Jiang, F., and Li, J., "Homotopic Approach and Pseudospectral Method Applied Jointly to Low Thrust Trajectory Optimization," Acta Astronautica, Vol. 71, February-March 2012, pp. 38-50.

${ }^{16}$ Bryson, A. and Ho, Y., Applied Optimal Control: Optimization, Estimation, and Control, Hemisphere, 1975, Chapter 2, pp. 65-69.

${ }^{17}$ Geering, H., Optimal Control with Engineering Applications, Springer Verlag, 2007, pp. 23-66.

${ }^{18}$ Battin, R., An Introduction to the Mathematics and Methods of Astrodynamics, AIAA Education Series, 1999, Chapter 3, pp. 128.

${ }^{19}$ Schaub, H. and Junkins, J., Analytical Mechanics of Aerospace systems, Vol. 1, AIAA, 2003, Appendix F, pp. 693-696.

${ }^{20}$ Gurfil, P., Herscovitz, J., and Pariente, M., "The SAMSON Project - Cluster Flight and Geolocation with Three Autonomous Nano-satellites," 26th AIAA/USU Conference on Small Satellites, Salt Lake City, UT, USA, August 2012. 